Check for updates

Cite this: Chem. Commun., 2020, 56,2677

Received 6th January 2020,

Accepted 6th February 2020

DOI: $10.1039 / \mathrm{d} 0 \mathrm{cc} 00106 \mathrm{f}$

rsc.li/chemcomm

\section{Gold and hypervalent iodine(III): liaisons over a decade for electrophilic functional group transfer reactions}

\author{
Somsuvra Banerjee, (D) ${ }^{\text {ab }}$ Vivek W. Bhoyare ${ }^{c}$ and Nitin T. Patil (D) *c
}

\begin{abstract}
Over the last two decades, hypervalent iodine(III) reagents have evolved from being 'bonding curiosities' to mainstream reagents in organic synthesis, in particular, electrophilic functional group transfer reactions. In this context, gold catalysts have not only emerged as a unique toolbox to facilitate such reactions (especially alkynylations) but also opened new possibilities with their different modes of reactivities for other functional group transfer reactions (acetoxylations and arylations). This feature article critically summarizes hitherto all such Au-catalyzed electrophilic functional group transfer reactions with hypervalent iodine(III) reagents, emphasizing their mechanistic aspects.
\end{abstract}

\section{Introduction}

Hypervalent iodine compounds, ${ }^{1}$ such as $\lambda^{3}$-iodanes and $\lambda^{5}$-iodanes, have always fascinated chemists because their 'unique reactivities' share strong ties with their 'unique bonding characteristics'. In particular, the molecular orbitals in $\lambda^{3}$-iodanes (1) are delocalized over three centres in the linear X-I-Y bond and occupied by two electrons from the (axial) $5 \mathrm{p}$ orbital of iodine and one electron each from apical ligand $\mathrm{X}$ and $\mathrm{Y}$ (Scheme 1a). The presence of such a weak, highly polarized, and three-center-fourelectron (3c-4e) 'hypervalent ${ }^{2}$ bond $(\mathrm{X}-\mathrm{I}-\mathrm{Y})$ in $\lambda^{3}$-iodanes (1) successfully validates their special structural features and highly electrophilic reactivity pattern, especially electrophilic functional group transfer reactions. ${ }^{3}$ In this regard, the pioneering work by Beringer and co-workers has paved the way for the development of diaryliodonium salts (2a) and alkynyliodonium salts (2b), both acyclic $\lambda^{3}$-iodanes, as electrophilic aryl- and acetylene synthons, repectively. ${ }^{4}$ This was an important milestone because iodonium salts (2) were able to deliver alkyne or aryl moieties 'directly' in an umpolung fashion and thus tremendously expand the scope of acetylene or aryl transfer reactions beyond the corresponding well-established nucleophilic versions. ${ }^{5}$ Later on, more methods such as alkenylations, alkylations and halogenations emulated the electrophilic functionalization strategy. ${ }^{6}$ However, research in this area witnessed a decline after 1995 probably because the high reactivity of acylic $\lambda^{3}$-iodanes was accompanied by

\footnotetext{
${ }^{a}$ Division of Organic Chemistry, CSIR-National Chemical Laboratory, Dr Homi Bhabha Road, Pune-411008, India

${ }^{b}$ Academy of Scientific and Innovative Research (AcSIR), Ghaziabad-201002, India

${ }^{c}$ Department of Chemistry, Indian Institute of Science Education and Research

Bhopal, Bhauri, Bhopal-462066, India. E-mail: npatil@iiserb.ac.in
}

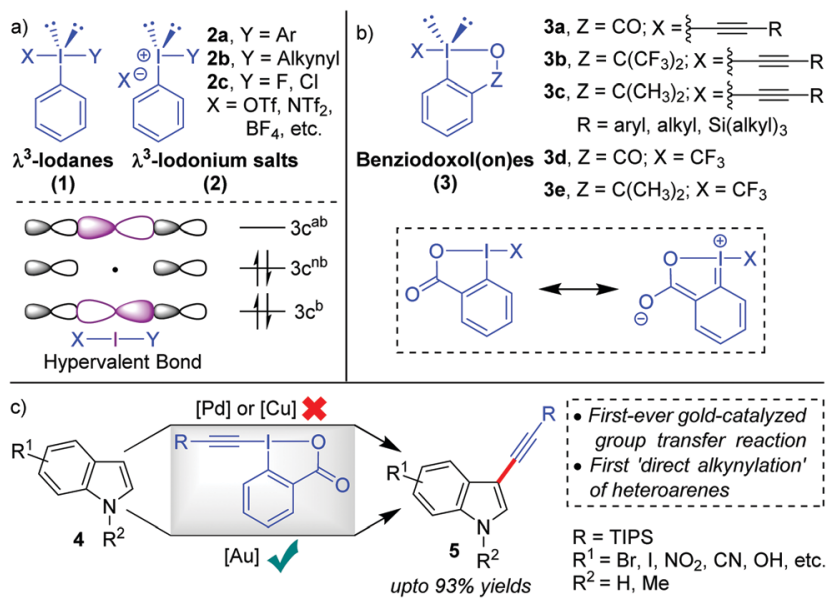

Scheme 1 (a) Structure and bondings in $\lambda^{3}$-iodanes and $\lambda^{3}$-iodonium salts, (b) benziodoxol(on)e (BX) analogues, and (c) C3-alkynylation of indoles: the first $\mathrm{Au}$-catalyzed electrophilic functional group transfer reaction.

instability (except for diaryliodonium salts $\mathbf{2 a}$ ) in the presence of strong bases or transition metals or during heating, which essentially limited their further applications. ${ }^{7 a}$ Therefore, striking a proper balance between reactivity and stability was necessary for future advancements in the field of electrophilic functional group transfer reactions.

With the inception of ethynylbenziodoxol(on)es (EBXs, 3a-c $)^{6 a, 7}$ in 1991, by the group of Ochiai and Shiro, ${ }^{8}$ the stability issue was mitigated ${ }^{9}$ by bridging the apical and the equatorial positions in a 5-membered heterocycle which introduces rigidity into the backbone (Scheme $1 \mathrm{~b}$ ). In cases where $\mathrm{Z}=\mathrm{CO}$, the stability was even more pronounced because of the 'extended' conjugative overlap between the lone pairs of electrons on the 
iodine atom and the $\pi$-orbitals of the aromatic nucleus. ${ }^{8}$ Later in 1996, the Zhdankin group developed a robust scheme for the synthesis of EBXs. ${ }^{10}$ Along this line, the greater stability of benziodoxol(on)es (BXs) enabled the preparation and isolation of otherwise unstable iodine(III) derivatives with I-CN, I-OOR, $\mathrm{I}-\mathrm{N}_{3}$, etc., all of which found practical application as the reagents for oxidative functionalization of organic molecules. ${ }^{1 e-i, 3}$ However, the first significant development, regarding the application of benziodoxol(on)e (BX)-based hypervalent iodine(III) reagents in mainstream organic synthesis, was the use of benziodoxol(on)ederived reagents $\mathbf{3 d}$ and $\mathbf{3 e}$ for electrophilic trifluoromethylation, by Togni and co-workers in 2006, which became an indispensible method to introduce the $-\mathrm{CF}_{3}$ functionality in the molecule. ${ }^{11}$ Next, in 2009, the Waser group successfully carried out the challenging alkynylations of indoles (4) with $\mathbf{3 a}$ in excellent yields (up to 93\%) and thus practically rejuvenated the field of electrophilic alkynylation (Scheme 1c). ${ }^{12,13}$ Usually, in such oxidative transfer reactions from hypervalent iodine(III) species, palladium and copper are the metals of choice because their well-established two-electron redox transformations enable swift oxidative addition of I(III)-X or I(III)-Y bonds to the lowvalent metal centre. ${ }^{5 b, 11,14}$ Copper can also initiate SET-induced radical fragmentation to formally transfer $\mathrm{X}^{(+)}$or $\mathrm{Y}^{(+)}{ }^{6 b, 11}$ In contrast, the key to success for these high-yield alkynylations with EBXs was the application of homogeneous Au-catalysis ${ }^{15}$ that exploited the nature of Au-catalysts (AuCl in this case) as tunable soft $\pi$-acids to activate the alkynyl unit installed in EBXs towards intermolecular attack by nucleophiles. In Waser's own words, "AuCl was uniquely able to activate TIPS-EBX for the C3-alkynylation of indole". ${ }^{7 b}$

There is indeed a reasonable explanation as to why the application of such cyclic $\lambda^{3}$-alkynyliodanes such as EBXs in electrophilic alkynylation took close to two decades to realize although their synthesis was already described in 1991. This is because the superiority of $\mathrm{Au}(\mathrm{I})$-catalysts in carbophilic activation over other late transition metals $(\mathrm{Cu}, \mathrm{Ag}, \mathrm{Pd})$ was understood only around the beginning of the 21st century. ${ }^{16}$ By the year 2009, exploration of gold's excellent capability for $\pi$-activation had gained pace. ${ }^{16}$ Conversely, the $\mathrm{C}-\mathrm{H}$ alkynylation of electron-rich heteroaromatics, which was going to be the first-ever synthetic application of EBXs, returned disappointing results for the Waser group with Pd- and Cu-catalysis (Scheme 1c). ${ }^{12,17}$ It was at this crucial juncture that the AuCl catalyst was successfully evaluated for the same, which marked the beginning of a new chapter in electrophilic functional group transfer reactions with hypervalent iodine(III) reagents, building upon the liaison between Au-catalysts and EBXs. In the following years, the Waser group was able to extend the scope of electrophilic $\mathrm{C}-\mathrm{H}$ alkynylation to a range of other electron-rich heterocycles, such as pyrroles, ${ }^{12,13}$ thiophenes, ${ }^{18}$ anilines, ${ }^{19}$ furans ${ }^{20}$ and benzofurans ${ }^{21}$ and thus established EBXs as 'general' electrophilic acetylene synthons. Since our group had been exclusively committed towards developing novel synthetic methodologies powered by Au-catalysis, ${ }^{22}$ we were able to contribute significantly to this field of Au-catalyzed alkynylations with EBXs.

As part of our continuous involvement with Au-catalysis, herein, we would like to present an overview of the fascinating, decade-long journey of Au-catalysts and hypervalent iodine(III) reagents. Building on a mechanistic perspective, the review intends to demonstrate how the uniqueness of Au-catalysts has made possible a myriad of electrophilic functional group transfer reactions such as alkynylations, arylations and acetoxylations with the use of hypervalent iodine(III) reagents over the last decade.

\section{Gold-catalyzed alkynylations with hypervalent iodine(III) reagents}

The current prominence of Au-catalysis in the field of hypervalent iodine(III) chemistry is majorly caused by the excellent liaison between $\mathrm{Au}$ and EBXs. To better understand this liaison, it is important to put a mechanistic perspective first, which governs such alkynylation reactions. In addition to their $\pi$-activation capability, Au-catalysts can also be 'forced' to switch between the +1 and +3 oxidation states under homogeneous catalysis conditions, thereby accessing a $\mathrm{Au}(\mathrm{I}) / \mathrm{Au}(\mathrm{III})$ catalytic cycle which delivers cross-coupled ${ }^{23}$ products. $^{22 a, 24}$ So far as the mechanism of Au-catalyzed alkynylations with EBXs is concerned, different scenarios have been discussed and a mechanistic ambiguity persisted for quite some time. ${ }^{13}$ To illustrate this, let's consider the case of Au-catalyzed C3-alkynylation of indoles (Scheme 2). The prevailing notion was that $\pi$-activation of the alkyne embedded in EBXs 3a triggers the addition of electron-rich (hetero)aromatic nucleus indole (Friedel-Crafts-type reaction) and leads to the formation of vinylgold(I) complexes (7) which, after a FritschButtenberg-Wiechell-type rearrangement (an $\alpha$-elimination/1,2shift sequence), produces the triple bond and the desired alkynylated product 5 (Scheme 2, cycle 1). However, researchers encountered some technical difficulties in garnering conclusive proof in favour of the $\pi$-activation mode. For instance, 1,2-silyl shift, which could have been one such conclusive proof, was rejected by ${ }^{13} \mathrm{C}$-labeling studies, whereas an alternative possibility of 1,2-indole shift could not be probed (Scheme 2b). ${ }^{12}$ Further, ligated $\mathrm{Au}(\mathrm{I})^{(+)}$complexes were not effective for such $\mathrm{C}-\mathrm{H}$ alkynylations due to strong poisoning by coordination to the benzoate group of the iodine(III) reagent. ${ }^{25}$ This left $\mathrm{AuCl}$ to be the sole optimum catalyst for alkynylations, which posed additional hurdles ${ }^{13}$ towards resolving the ambiguity.

A modified version of the $\pi$-activation mode was introduced by Ariafard's group who employed DFT studies to suggest that the iodine(III) center in EBXs acts as a Lewis acid for activating the alkyne, even more efficiently than the Au(I)-center ${ }^{25}$ (Scheme 2, cycle 2). The catalytic reaction starts with the coordination of the alkyne moiety of the iodine(III) reagent to the AuCl catalyst to generate $\mathbf{6}$, followed by transfer of the alkynyl group from I(III) to $\mathrm{Au}(\mathrm{I})$ to access a less stable I(III)-activated alkyne species, 9, which remains in equilibrium with $\mathbf{6}$. Thereafter, the nucleophilic attack of indole on the I(III)-activated alkyne 9 gives a vinylgold(I) complex, 10, which is the rate-determining step as per the calculations. Complex 10 then undergoes $\beta$-elimination to give the desired product 5, regenerating AuCl.

Among these mechanistic conundrums, two other modes of activation regarding Au-catalyzed cyclizations with EBXs were 
a)
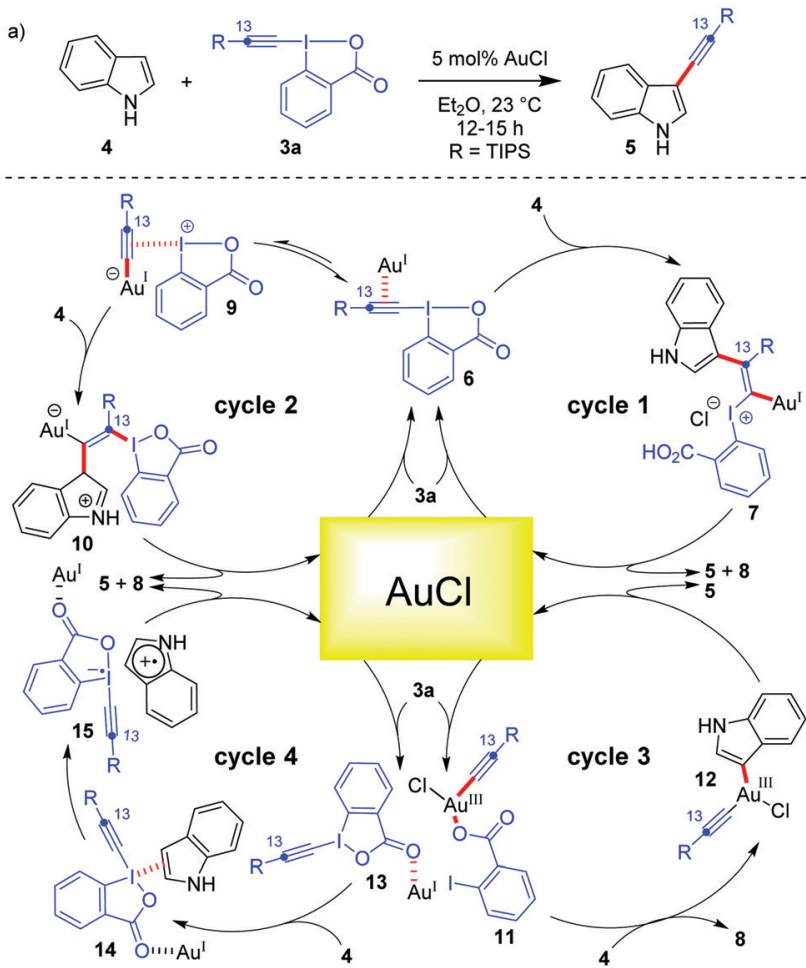

cycle 1: $\pi$-activation mode

cycle 3: $\mathrm{Au}(\mathrm{I}) / \mathrm{Au}(\mathrm{III})$ catalysis mode cycle 2: $\pi$-activation mode (Ariafard's model) cycle 4: Lewis acid activation/SET mode

b)

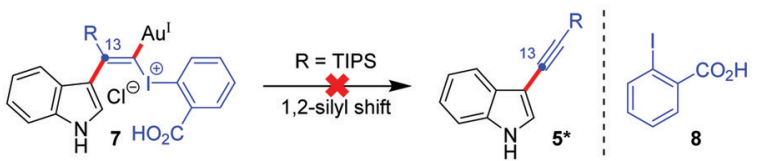

Scheme 2 (a) Different mechanistic possibilities depicted for C3-alkynylation of indoles; and (b) ${ }^{13} \mathrm{C}$ labelling studies to probe any 1,2-silyl shift.

also speculated. ${ }^{13}$ In the $\mathrm{Au}(\mathrm{I}) / \mathrm{Au}(\mathrm{III})$ catalysis mode, the $\mathrm{Au}(\mathrm{I})$ species is oxidized to form a $\mathrm{Au}(\mathrm{III})$-complex, 11, followed by indole metalation and reductive elimination to furnish the desired alkynylated product (Scheme 2, cycle 3). In the Lewis acid activation/SET mode, AuCl acts as a Lewis acid and increases the electrophilicity of the iodine atom (Scheme 2, cycle 4). The activated hypervalent iodine $\mathbf{1 3}$ forms a charge transfer complex, 14, with the electron-rich indole. Thereafter, the SET process takes over and subsequently produces the desired alkynylated product 5. Though the Lewis acid activation/SET mode (Scheme 2, cycle 4) was dismissed at the earliest by the Waser group by a series of control experiments, ${ }^{13}$ the distinction between the $\pi$-activation mode (cycle 1 or 2 ) and the $\mathrm{Au}(\mathrm{I}) / \mathrm{Au}(\mathrm{III})$ catalysis mode (cycle 3 ) for Au-catalyzed alkynylations became arduous. In this context, based on the computational study by the Ariafard group ${ }^{26}$ on the Au-catalyzed domino cyclization/ alkynylation approach ${ }^{20}$ to 3-alkynyl furans from keto-allenes, two very important rationales can be given regarding the general mechanism of alkynylation:

(A) If the yields of the reaction concerned are below par with 'naked' Au(I)- or Au(III)-catalysts (typical $\pi$-activation), it is likely that the reaction 'demands' a $\mathrm{Au}(\mathrm{I}) / \mathrm{Au}(\mathrm{III})$ catalysis mode. To serve this purpose, a $\mathrm{Au}(\mathrm{III})$-stabilizing ligand in the reaction (e.g. $\mathrm{N}$ - and $\mathrm{O}-$ coordination of picolinic acid in this case) needs to be introduced, which has been observed to improve the yields substantially.

(B) In such cases, another mode of reactivity is also feasible which involves the interplay of both the reactivities (i.e., both $\pi$-activation and $\mathrm{Au}(\mathrm{I}) / \mathrm{Au}(\mathrm{III})$ catalysis: the 'interplay' mode).

Both rationales $\mathrm{A}$ and $\mathrm{B}$ were later substantiated with experimental evidence by the groups of Hashmi ${ }^{27}$ and Patil ${ }^{28}$ in their direct alkynylation of cyclopropenes and 1,2-oxyalkynylation of $\mathrm{N}$-allenamides, respectively. Therefore, the Au-catalyzed alkynylations with EBXs discussed herein are categorized into three broad classes based on the activation mode of Au-catalysts.

1. Alkynylations via the $\pi$-activation mode in which $\mathrm{Au}(\mathrm{I})$ catalysts activate the alkyne system of EBXs or that of the partner nucleophile.

2. Alkynylations via the $\mathrm{Au}(\mathrm{I}) / \mathrm{Au}(\mathrm{III})$ catalysis mode where EBX oxidizes $\mathrm{Au}(\mathrm{I})$ to $\mathrm{Au}(\mathrm{III})$ in situ and the final alkynylated product results from a reductive elimination from the alkynylgold(III) intermediate.

3. Alkynylations via the 'interplay’ mode wherein Au-catalysts activate the $\pi$-system embedded in the partner nucleophile and are also oxidized to Au(III) by EBXs (not necessarily in that order). Further, the final alkynylated product results from a reductive elimination from the alkynylgold(III) intermediate.

The reports are segregated according to the mechanism proposed originally by the authors unless new mechanistic insights came to the fore. Furthermore, if we have assigned the report to a particular category according to the original mechanism but sincerely believed that the actual mechanism might differ based on our rationale $\mathrm{A}$, we have included our perspective there. On grounds of the same rationale $\mathrm{A}$, in case of unresolved mechanistic ambiguities mentioned in the article concerned between a $\pi$-activation mode and a $\mathrm{Au}(\mathrm{I}) / \mathrm{Au}(\mathrm{III})$ catalysis mode, it is categorized as a $\pi$-activation mode.

\subsection{Alkynylations via the $\pi$-activation mode}

Theoretical studies by the Ariafard group ${ }^{25}$ currently suggest that the C3-alkynylation of indoles (4) leading to 5 follows cycle 2 (Scheme 2), which, a priori, also corroborates with our rationale A that no $\mathrm{Au}(\mathrm{III})$-stabilizing factor (ligand coordination in TS) is required in the reaction to increase the yield to the desired level. Hence, C3-alkynylation of indoles is categorized as 'alkynylations via the $\pi$-activation mode'. In fact, all the Au-catalyzed alkynylations with EBXs following the $\pi$-activation mode should, in principle, follow cycle 2, Scheme 2 . However, while describing this section, we have considered the mechanisms originally proposed by the authors.

Further, there were subsequent improvements and modifications being reported for the construction of C3-alkynylated indoles (5). First, the original Au-catalyzed, direct $\mathrm{C}-\mathrm{H}$ alkynylation of indoles was made more efficient by the Bolm group by conducting the reaction under mechanochemical conditions (Scheme 3a). ${ }^{29}$ In comparison to Waser's method, Bolm's ball milling method at $30 \mathrm{~Hz}$ sports a solvent-free approach, a lower AuCl-loading (2 mol\% vs. $5 \mathrm{~mol} \%$ ) and a shorter reaction (milling) time (1.5 h vs. $12 \mathrm{~h}$ ), although the yields of alkynylated 

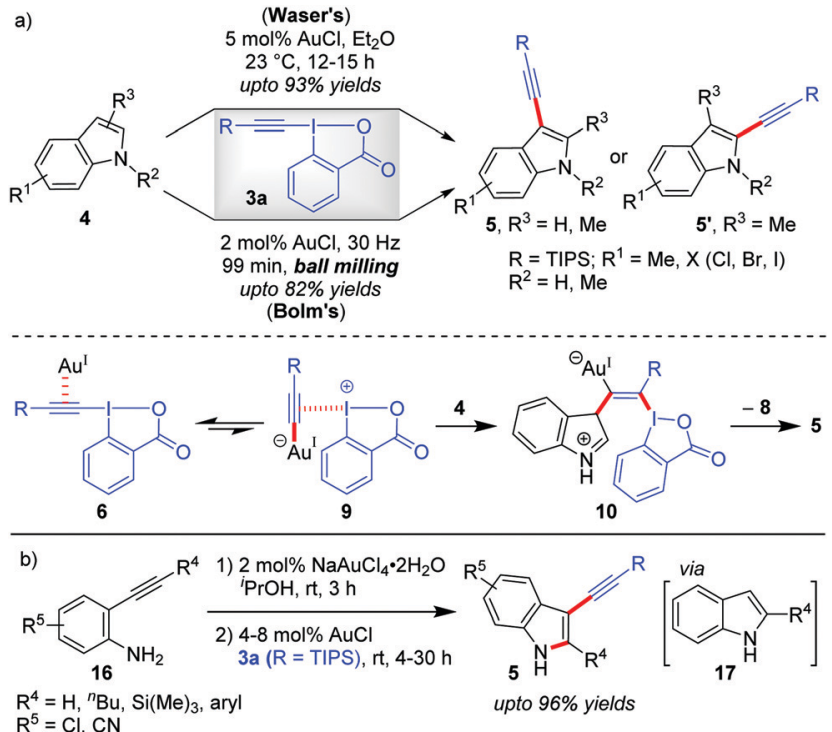

Scheme 3 (a) Comparison of Waser's and Bolm's methods for C3-alkynylation of indoles; and (b) one-pot sequential route to 2-substituted-3-alkynylindoles from unprotected ortho-alkynylanilines.

products were moderate (up to $82 \%$ ). Later on, the Waser group combined the $\mathrm{Au}(\mathrm{III})$-catalyzed cyclization of 2-alkynylanilines (16) with the $\mathrm{Au}(\mathrm{I})$-catalyzed $\mathrm{C} 3$-selective direct alkynylation of indoles (4) using EBXs (3a) in a one-pot sequential fashion to give straightforward access to 2-substituted-3-alkynylindoles (5) (up to $96 \%$ yields, Scheme $3 \mathrm{~b}$ ). ${ }^{30}$ This remains to be the first example of a one-pot process combining a $\mathrm{Au}(\mathrm{III})$-catalyst and a $\mathrm{Au}(\mathrm{I})$-catalyst. Of note, $\mathrm{AuCl}$ was unable to catalyze step 1 of the sequential process due to the 'stochastical' degradation of AuCl under these conditions and $\mathrm{NaAuCl}_{4}$ was observed to fail the alkynylation step.

After the maiden success of the Au-catalyzed $\mathrm{C}-\mathrm{H}$ alkynylation of electron-rich heteroarene indoles (Scheme 2), ${ }^{12,13}$ the Waser group further extended the scope of direct alkynylation reaction to other heteroarenes like pyrroles, ${ }^{12,13}$ thiophenes ${ }^{18}$ and benzofurans ${ }^{21}$ (19a-19d, Scheme 4). While the same reaction conditions leave unsatisfactory outcomes in the case of less electron-rich heteroarenes such as benzofurans and thiophenes, the activation of EBXs (3a) with either TFA or $\mathrm{Zn}(\mathrm{OTf})_{2}(1: 1$ ratio with respect to EBXs) proved to be a highly successful strategy. The role of such Lewis acid/Brønsted acid is supposedly to activate the EBXs by complexing with the carboxylate group of the hypervalent iodine(III) reagent, enhancing their electrophilic reactivity for efficient acetylene transfer. Along this line, further modulation of the reactivity of EBXs via trans-influence ${ }^{31}$ of ligands in the hypervalent $\mathrm{X}-\mathrm{I}-\mathrm{Y}$ bond and substituent effects on the aromatic ring were also studied by the Waser group. ${ }^{13}$ In the same report, the low-yield issue in the case of alkynylation of pyrroles (18a) was also addressed by using stoichiometric amounts of pyridine as an additive. The pyridine was able to suppress the competitive degradation of pyrroles by removing adventitious $\mathrm{HCl}$ generated from AuCl during the reaction. However, use of excess EBX led to dialkynylation of pyrroles.

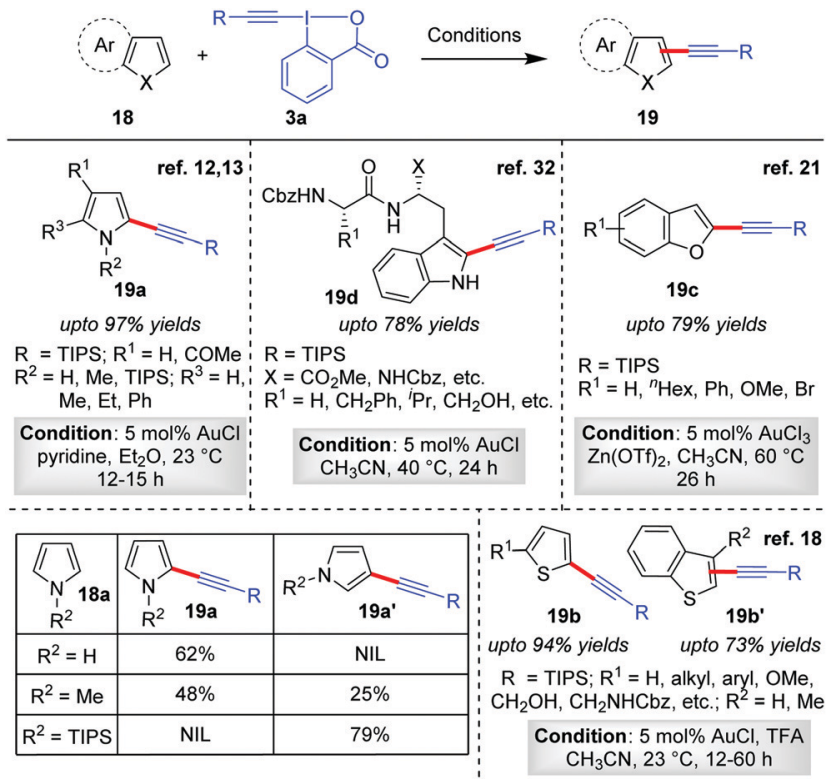

Scheme 4 Alkynylation of electron-rich heterocycles: pyrroles, thiophenes, benzofurans and tryptophan.

For all the heteroarenes, the regioselectivity of the alkynylation reaction happens to be in exclusive agreement with electrophilic aromatic substitution $\left(\mathrm{S}_{\mathrm{E}} \mathrm{Ar}\right)$; that is, it is reliant on the relative electron richness of the respective sites. The regioselectivity can also be predicted by considering the thermodynamic stability of the intermediates obtained by the Friedel-Crafts addition of heteroarenes onto intermediate $\mathbf{9}$ (Scheme 3a). In case the site concerned is occupied, the alkynylation would proceed to the next available site. For instance, the formation of C2-alkynylated products $\left(5^{\prime}\right)$ were observed exclusively for C3-substituted indoles (Scheme 3a), whereas for pyrroles the regioselectivity was sensitive to the steric bulk on the nitrogen atom ${ }^{12}$ (Scheme 4, table). Therefore, by applying easily removable protecting groups such as TIPS, the regioselectivity could be easily controlled. The robustness of the $\mathrm{C}-\mathrm{H}$ alkynylation was further demonstrated in the direct alkynylation of tryptophan in peptides (19d, up to $78 \%$ yields, Scheme 4$),{ }^{32}$ which is indeed highly useful for bio-conjugating tryptophan under mild conditions without the installation of non-natural amino acids. Of note, the mechanism for obtaining alkynylated products 19a-19d follows cycle 1 , Scheme 2, as originally proposed by the authors.

It was quite obvious from alkynylation of heteroarenes that the electronic effects play a major role in determining site selectivity. In particular, the directing effect of the heteroatom functional group (e.g. 'NR') is the most effective amongst all, functioning as an embedded enamine-type system. Along this line, the Patil ${ }^{33}$ group and the Li group, ${ }^{34}$ in 2016, independently reported a Au-catalyzed site-selective C4-alkynylation of isoquinolones/2-pyridones (20), respectively, giving 21 (up to 92\% yields, Scheme 5). As usual, the intrinsic electronic bias of the isoquinolones/2-pyridone rings allowed the alkynylation at the most electron-rich $\mathrm{C} 4$ site. A theoretical investigation on the operating mechanism in this reaction by the Yu and Liu group ${ }^{35}$ reinforced 


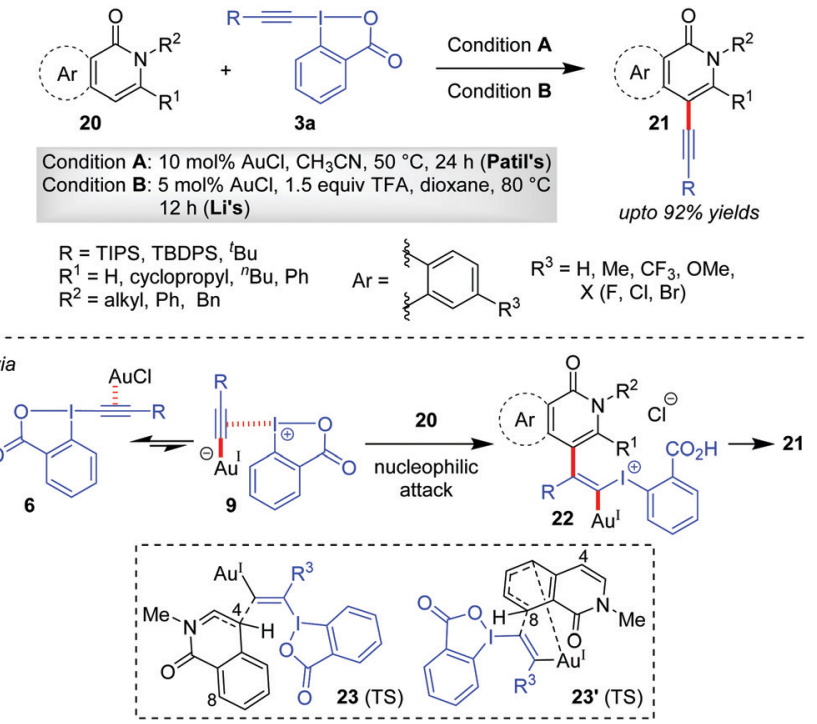

Scheme 5 Alkynylation of isoquinolones and 2-pyridones at the C4 site.

Ariafard's proposal ${ }^{25}$ that the iodine(III) centre, instead of the $\mathrm{Au}(\mathrm{I})$-centre, acts as a strong Lewis acid to activate the alkyne moiety efficiently ( $c f$. 11). In addition, they reasoned that the C4-alkynylation product is preferred over the C8-alkynylation product for isoquinolone systems mainly because of the higher steric effects in $23^{\prime}$ (TS) compared with that in $\mathbf{2 3}$ (TS). Of note, the reactions in Scheme 5 were originally a part of catalystcontrolled regiodivergent alkynylation programs featuring $\mathrm{Au}(\mathrm{r})-$ and $\mathrm{Rh}(\mathrm{III})$-catalysts. It is the coordination with the amide carbonyl group that directs $\mathrm{Rh}$ (III)-catalysts to effect $\mathrm{C}-\mathrm{H}$ activation at a different site.

Alkynylation promoted by pre-formed acyclic enamine systems was first evaluated by the Patil group where tandem alkynylation of ortho-hydroxyarylenaminones (24) followed by intramolecular cyclization was performed to generate a diverse array of 3-alkynylchromones (25, up to $96 \%$ yields, Scheme 6). ${ }^{36}$ Control experiments suggested that enaminone $\mathbf{2 4}$ would undergo alkynylation first to produce intermediate $\mathbf{2 7}$ (via 26) which after intramolecular
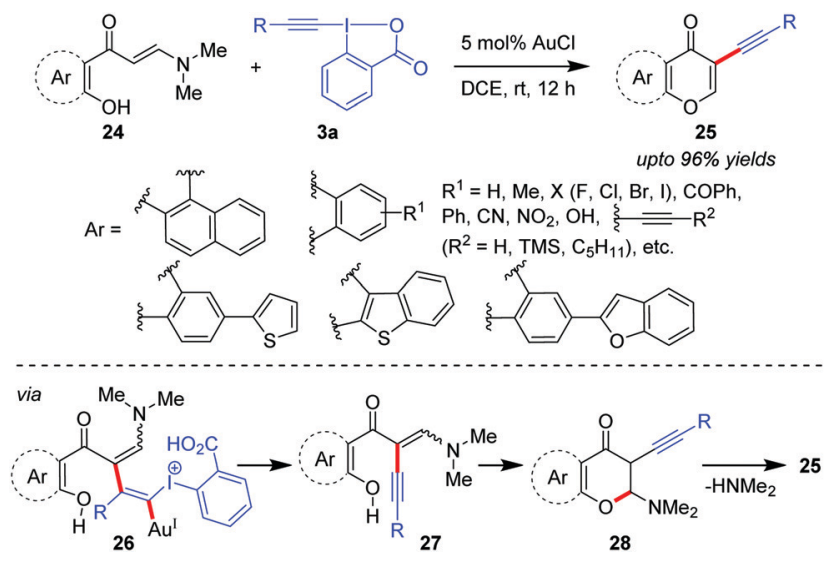

Scheme 6 Alkynylation/cyclization cascade of ortho-hydroxyarylenaminones to 3-alkynylchromones. cyclization would afford intermediate 28. Finally, the spontaneous loss of $N, N$-dimethylamine would occur to generate 3-alkynylchromones 25.

Exercising cooperation between amine catalysis and Au-catalyzed alkynylation via EBXs, the Huang group disclosed the first direct $\alpha$-vinylidenation of aliphatic aldehydes 29 (Scheme 7a). ${ }^{37}$ The authors reasoned that the addition of enamine 33 to the Au-activated $\pi$-system in EBXs (3a) would be followed by an $\alpha$-elimination/1,2-shift to lead to 34 which, after tautomerization, gives the key ynenamine intermediate 35 . Intermediate 35 would be further hydrolyzed by $\gamma$-protonation to yield $\alpha$-allenyl aldehydes (30). The competing $\alpha$-protonation, which leads to $\alpha$-alkynyl aldehydes (31), was assumed to be less favoured by the authors. Unfortunately, the selectivity of the reaction towards $\alpha / \gamma$-protonation was not satisfactory enough and the two constitutional isomers $\mathbf{3 0}$ and $\mathbf{3 1}$ were observed to be inseparable (combined yields up to $88 \%$ ). The methodology can be further extended to affect a relay ${ }^{38} \alpha$-vinylidenation $/ \gamma$-alkynylation of aldehydes 29 with excess equivalents of EBXs through a subsequent second electrophilic alkynylation of the ynenamine intermediate $\mathbf{3 5}$ preferably at the $\gamma$-carbon atom (Scheme $7 \mathrm{~b}$ ). The obtained $\gamma$-alkynyl allenyl aldehydes (37, up to $79 \%$ yields) were rather unstable and were smoothly converted into 2-alkynyl-3-silylfurans (38, up to $93 \%$ yields) using $\mathrm{AuCl}_{3}$, thus rendering a highly efficient two-step synthesis of trisubstituted furans $\mathbf{3 8}$ from aldehydes 29. Further, an attempt towards developing the enantioselective version of the one-pot $\alpha$-vinylidenation/ $\gamma$-alkynylation cascade with a chiral primary amine, 36, led to $17 \%$ ee of the resulting allene 37 .
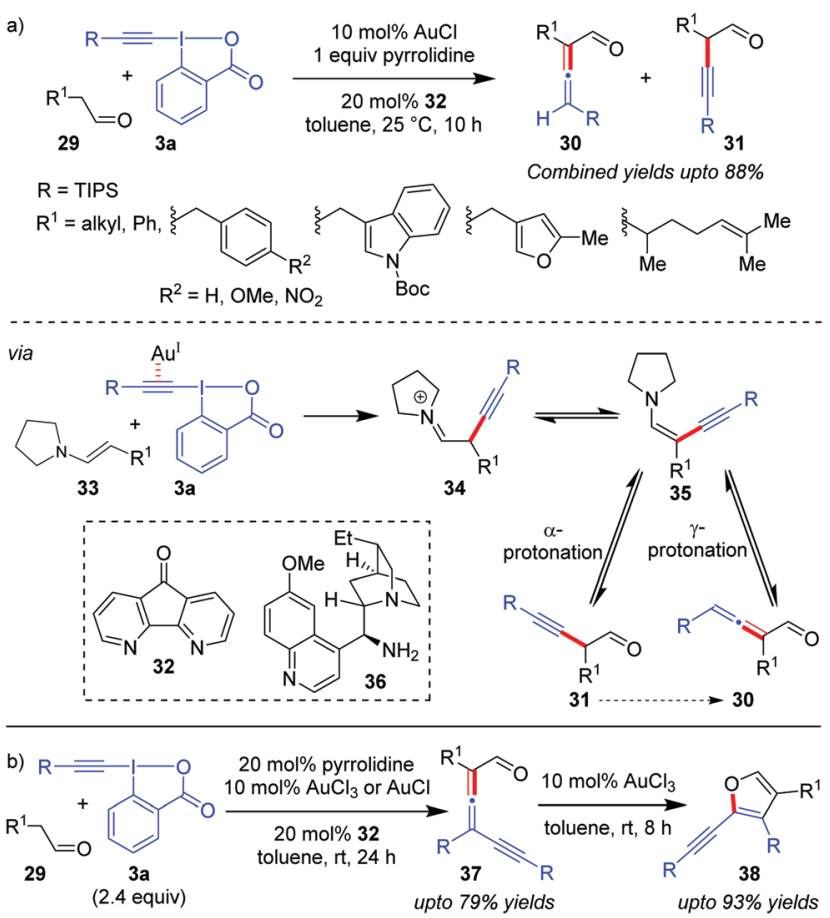

Scheme 7 (a) $\alpha$-Vinylidenation of aliphatic aldehydes using a Au/pyrrolidine catalyst system; and (b) one-pot relay of $\alpha$-vinylidenation $/ \gamma$-alkynylation of aliphatic aldehydes. 

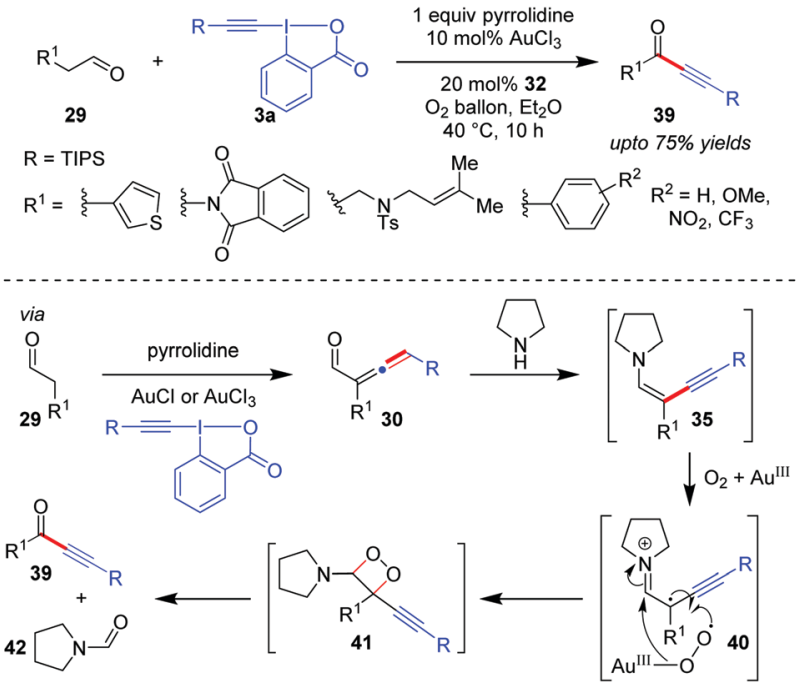

Scheme 8 Direct access to ynones from aldehydes via oxidative C-C bond cleavage of intermediate ynenamine under aerobic conditions.

An interesting extension to the Huang group's $\alpha$-vinylidenation can be seen in their later work where ynenamine 35, generated in situ from the $\alpha$-allenyl aldehyde $\mathbf{3 0}$ and the secondary amine pyrrolidine, can undergo a subsequent in situ oxidative cleavage of the $\mathrm{C}-\mathrm{C}$ bond with aerobic molecular oxygen to furnish ynones 39 (up to $75 \%$ yields, Scheme 8 ). ${ }^{39}$ This became the first direct synthesis of various ynones $\mathbf{3 9}$ from readily available aldehydes 29. The isolation of the byproduct pyrrolidine-1-carbaldehyde 42 in good yield supports the assumption that the aerobic oxidation of 35 occurs through the 1,2-dioxetane intermediate 41. Further, since this oxidative cleavage step demands the presence of a Au-catalyst and adopts a free radical pathway as evidenced by control experiments, it is surmised that $\mathrm{Au}(\mathrm{III})$ activates molecular oxygen and facilitates single electron transfer from the electron-rich ynenamine 35 to $\mathrm{O}_{2}(c f$. 40).

Similar to the formation of ynenamine 35 from aliphatic aldehydes 29 by the action of a Au/secondary amine catalyst system, Huang's group in 2018 isolated ynedienamines 44 from ynones 43 in a near-quantitative yield (Scheme 9). ${ }^{40}$ Products 44

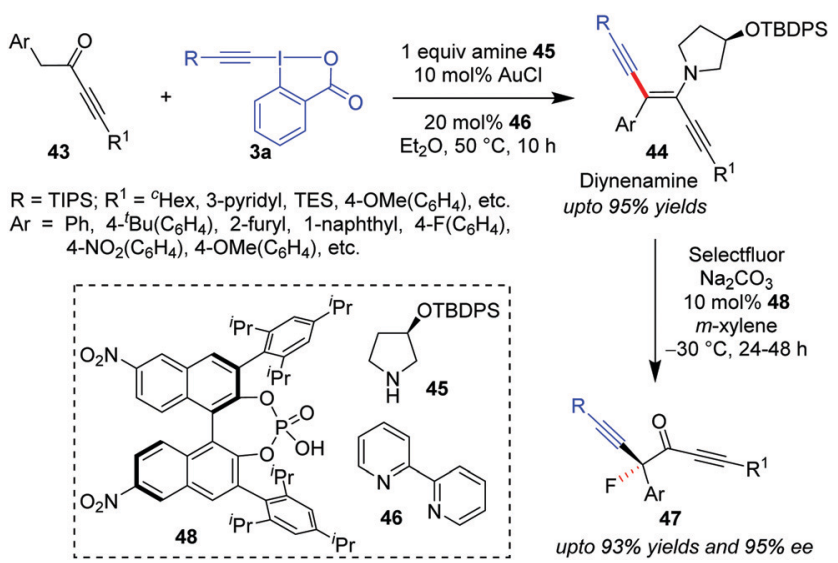

Scheme 9 Synthetic protocol for asymmetric $\alpha$-difunctionalization of ynones. were subsequently involved in an asymmetrical fluorination by Selectfluor/chiral phosphoric acid (48), yielding enantioenriched ynones 47 in high yields (up to 93\%), boasting an $\alpha$-quaternary carbon which contains a fluorine atom. To this end, the current methodology became the first streamlined synthetic protocol for asymmetric $\alpha$-difunctionalization of ynones. Herein, the secondary amine was a recyclable chiral amine auxiliary, 45, which, in combination with 48, eventually increased the enantioselectivity of the fluorination step.

The reaction conditions for $\mathrm{C}-\mathrm{H}$ alkynylation of heteroarenes were also well tolerated for electron rich arenes like anilines (49) and 1,3,5-trimethoxybenzene to obtain 50 (up to $85 \%$ yields) and 51 (50\%), respectively (Scheme 10). ${ }^{19}$ The reaction was observed to be para-selective, which is complementary to the existing directing group-assisted transition metal-catalyzed ortho-alkynylation ${ }^{41}$ of anilines 49 and the mechanism is similar to cycle 1 of Scheme 2, as originally proposed by the authors. With para-substituted 4-methyl- $N, N$-dimethyl aniline (49a), C( $\left.\mathrm{sp}^{3}\right)-\mathrm{H}$ alkynylated products (52) were obtained as the major product $(22 \%)$ due to the relatively high oxidation capability of $\mathbf{3 a}$ with traces of orthoalkynylated products (53).

Azulenes (54), whose special [7+5] membered aromatic ring system contributes to the dipolar nature of the molecule with a high dipole moment $(1.08 \mathrm{D})$ and hence increased reactivity, were efficiently alkynylated at the C3 site to obtain 55 (up to $79 \%$ yields) by the Novák group using Au-catalyzed alkynylation with EBXs (Scheme 11). ${ }^{42}$ However, in cases where $\mathrm{R}_{2}=\mathrm{H}$,
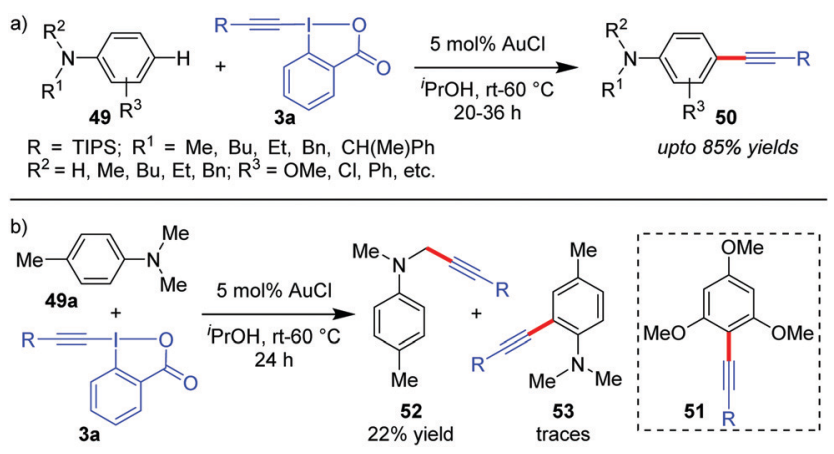

Scheme 10 para-Selective direct alkynylation of anilines.

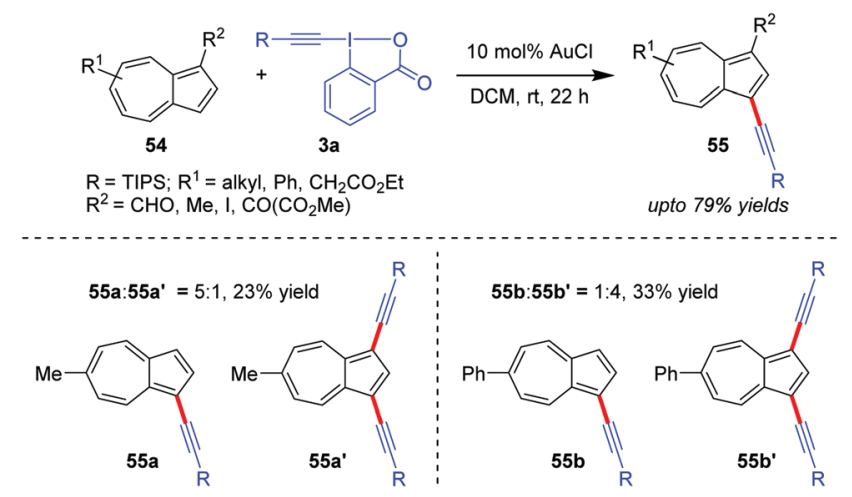

Scheme 11 Direct alkynylation of azulenes. 


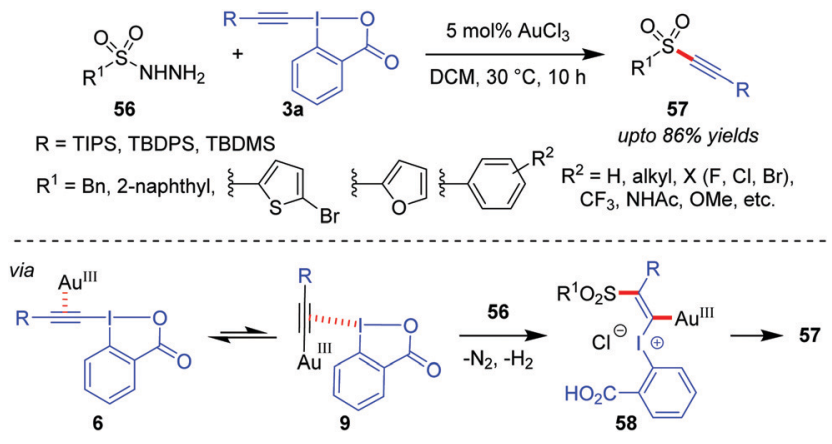

Scheme 12 Dehydrazinative C(sp)-S cross-coupling reactions of arylsulfonyl hydrazides.

a mixture of the corresponding mono- and dialkynylated products is formed ( $c f . \mathbf{5 5 a}: \mathbf{5 5 \mathbf { a } ^ { \prime }}$ and $\mathbf{5 5 b} \mathbf{5 5} \mathbf{b}^{\prime}$ ). The authors originally proposed the mechanism to be similar to cycle 1 of Scheme 2 .

$\mathrm{A} \mathrm{Au}(\mathrm{III})$-catalyzed dehydrazinative $\mathrm{C}(\mathrm{sp})-\mathrm{S}$ coupling reaction between 3a and arylsulfonyl hydrazides (56) was developed by the Patil group for the synthesis of a variety of alkynyl aryl sulfones (57, up to $86 \%$ yields, Scheme 12$).{ }^{43}$ The current reaction describes a unique way for the use of sulfonyl hydrazides as sulfur nucleophiles in Au-catalyzed reactions. Interestingly, the performance of $\mathrm{Au}(\mathrm{III})$-catalysts in the current alkynylation reaction is better than that of $\mathrm{Au}(\mathrm{I})$, which is generally uncharacteristic of such alkynylations.

Unlike the previous cases which involve the activation of only 3a, Patil's report on the Au-catalyzed aminoalkynylation of alkynes leading to the formation of quinalizinones 60 (up to $87 \%$ yields) from pyridinoalkynes 59 involves the activation of both $3 a$ and its coupling partner 59 (Scheme 13a). ${ }^{44}$ The vinylgold(I) complex 63 results from an intramolecular addition of a tethered nucleophile to the Au-activated $\pi$-system incorporated into 59. Thereafter, a direct nucleophilic attack of such organogold(I) intermediates $\mathbf{6 3}$ onto EBXs can also lead to the desired alkynylated products.

During control experiments, when 6-aryl-2-pyridone 64, obtained from 59a with conditions A, was subjected to the standard reaction conditions, the substrate underwent a 'direct' C3-alkynylation (65, Scheme 13b), analogous to the alkynylation previously developed by Patil $^{33}$ and $\mathrm{Li}^{34}$ This implies that a complementary reactivity can be developed out of these pyridinoalkynes (e.g., 59a), wherein the current domino cyclization/alkynylation approach (conditions B) would lead to C8-alkynylated product 60 a (87\% yield) and performing the same in a sequential manner (conditions $\mathrm{A}$ and then conditions B) would lead to a C3-alkynylated product, 65 (56\% yield over two steps). Of note, this report constitutes the standalone example of Au-catalyzed aminoalkynylation of alkynes.

\subsection{Alkynylations via the $\mathrm{Au}(\mathrm{I}) / \mathrm{Au}(\mathrm{III})$ catalysis mode}

Gold-catalyzed C-C cross-coupling reactions using hypervalent iodine(III) reagents as sacrificial oxidants have been existing for quite some time. ${ }^{45}$ But developing Au-catalyzed, sacrificial oxidant-free $\mathrm{C}-\mathrm{C}$ cross-coupling reactions ${ }^{24 i}$ requires a single entity OxC (67) that could serve a dual role as an oxidant and a coupling partner (Scheme 14a). EBXs (3a-c), by design, can

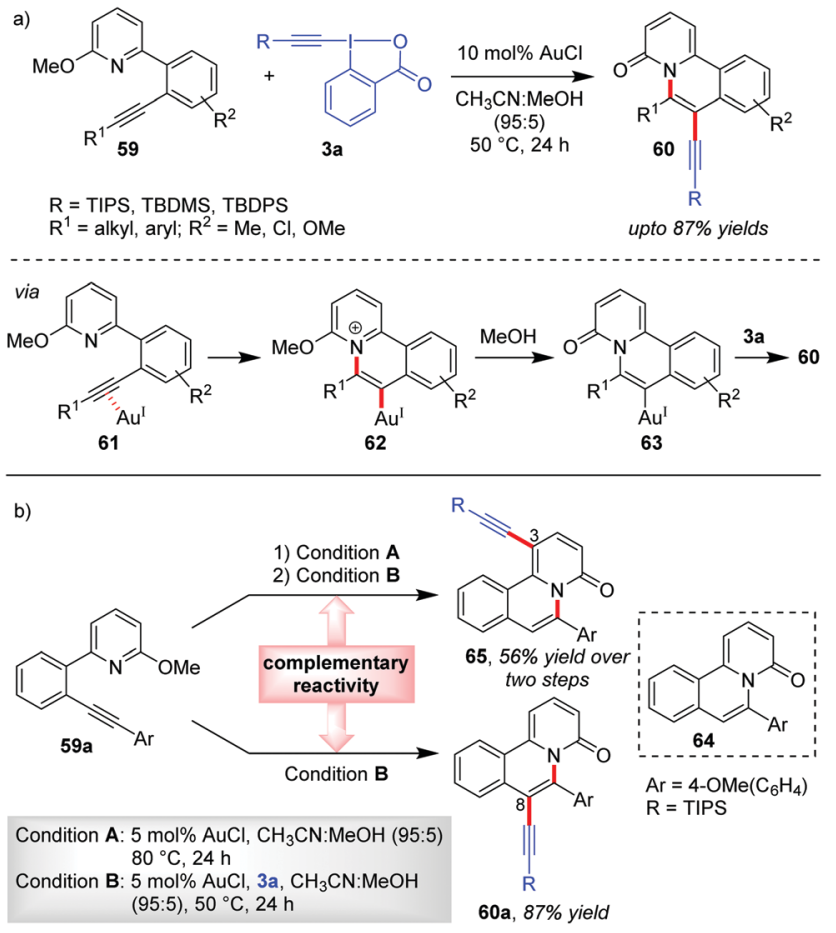

Scheme 13 (a) Aminoalkynylation of alkynes to access alkynylated quinalizinones; and (b) C3-alkynylation vs. C8-alkynylation: complementary reactivity of pyridinoalkynes.

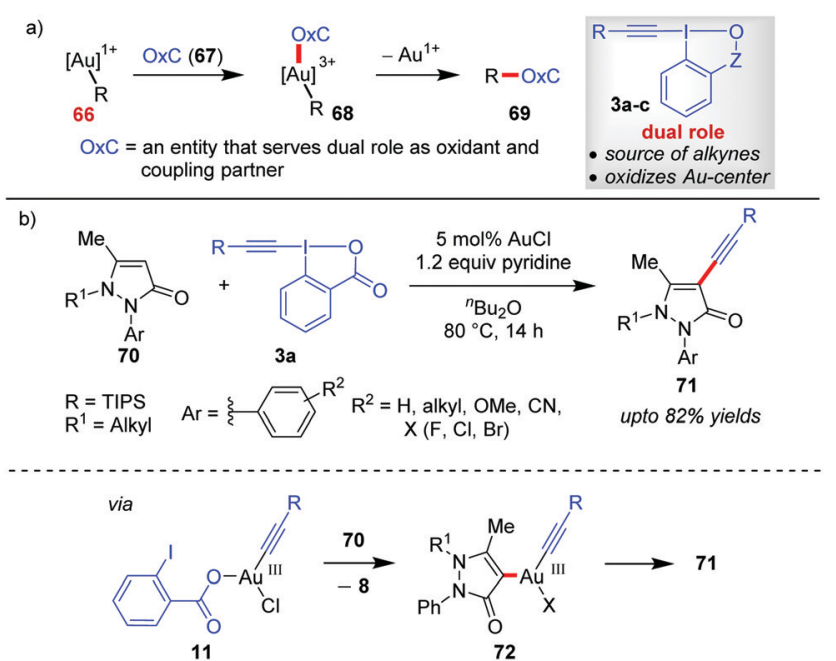

Scheme 14 (a) New coupling partner, 'Oxc' for executing Au-catalyzed, redox-neutral C-C cross-coupling; and (b) alkynylation of $N$-alkyl pyrazolone at the C4-site.

function as $\mathrm{OxC}(67)$ by giving researchers the opportunity to oxidize $\mathrm{Au}(\mathrm{I})$ ( $c f$. 66) to $\mathrm{Au}(\mathrm{III})(c f$. 68) in situ as well as the concomitant transfer of an alkynyl group. This premise of EBXs (3a) serving the dual role of an oxidant and an alkyne surrogate greatly assisted the development of Au-catalyzed, alkynylative $\mathrm{C}-\mathrm{C}$ cross-coupling reactions and is discussed in this section.

In continuation of the regiodivergent alkynylations as mentioned in the discussion pertaining to Scheme 5, the Xia group reported 
a $\mathrm{Au}(\mathrm{I})$-catalyzed alkynylation of 2-aryl-3-pyrazolones (70) at the most electron-rich $\mathrm{C} 4$ site to give 71 (up to $82 \%$ yields, Scheme 14). ${ }^{46}$ On the basis of the H/D exchange at the C4 position of the pyrazolone (70), the authors proposed an alkynylative cross-coupling pathway involving a $\mathrm{Au}(\mathrm{I}) / \mathrm{Au}(\mathrm{III})$ catalytic cycle where the oxidative addition of $\mathrm{Au}(\mathrm{I})$ with $3 \mathbf{a}$ leads to the formation of an alkynylgold(III) intermediate (11), which after an heteroarene metalation ( $c f$. 72) and reductive elimination affords the final product $\mathbf{7 1}$ and regenerates the $\mathrm{Au}(\mathrm{I})$-catalyst. However, the evidence in favour of the $\mathrm{Au}(\mathrm{I}) / \mathrm{Au}$ (III) catalytic cycle is apparently not concrete. As we shall see later in this Section (2.2) and Section 2.3, such a $\mathrm{Au}(\mathrm{I}) / \mathrm{Au}$ (III) catalytic cycle warrants the use of a weaker bidentate $\pi$-acceptor ligand like phen (76) as a $\mathrm{Au}(\mathrm{III})$ stabilizing agent, which plays an important role in promoting the oxidation of the Au(I)-complex by EBXs and facilitates a rapid reductive elimination from the $\mathrm{Au}(\mathrm{III})$-complex. Nevertheless, the role of pyridine acting as a ligand in facilitating oxidative addition to such a $\mathrm{Au}(\mathrm{I})$ complex cannot be neglected. ${ }^{27 b}$

In 2017, Au-catalyzed C(sp)-C(sp) cross-coupling of terminal alkynes 73 with alkynyl hypervalent iodine(III) reagents 3 to furnish unsymmetrical 1,3-butadiynes 75 was independently developed by Liu and Patil with up to $98 \%$ and $86 \%$ yields, respectively (Scheme 15). ${ }^{47}$ The initial sacrificial oxidantempowered, Au-catalyzed version of $\mathrm{C}(\mathrm{sp})-\mathrm{C}(\mathrm{sp})$ cross-coupling of two terminal alkynes developed by the Shi group ${ }^{48}$ overcame the selectivity issues posed by conventional $\mathrm{Pd}-/ \mathrm{Cu}-/ \mathrm{Ni}$-catalyzed cross-couplings (Glaser-Hay ${ }^{49}$ coupling and Cadiot-Chodkiewicz $^{50}$ coupling) via the ability of $\mathrm{Au}(\mathrm{I})$ to promote the preferential formation of arylgold acetylide (77) over alkylgold acetylide (78) for electronically-biased terminal alkynes (up to 93\% yields of $\mathbf{7 5}$, Scheme 15a). The current report is a reasonable modification to Shi's work where the use of EBXs, which can act as oxidants as well as alkyne transfer agents, not only eliminated the need for an external oxidant (e.g. PhI $\left.(\mathrm{OAc})_{2}\right)$ but also helped the preferential formation of one metal acetylide exclusively over the other without the need for electronic bias among the alkynes concerned. If we draw a comparison between the two sets of reaction conditions set by the respective groups, the principal shortcoming in Liu's reaction conditions, would be the use of a $\operatorname{Ag}(\mathrm{II})^{(+)}$additive and sub-stoichiometric amounts of ligand 76 (0.5 equiv.) to achieve the desired yield and heteroselectivity of the reaction, which is not the case with Patil's reaction conditions (no $\operatorname{Ag}(\mathrm{I})^{(+)}$, 0.15 equiv. of 76). Conversely, the substrate scope in Liu's work, as compared to that in Patil's, accommodates alkylEBXs (3b, $\mathrm{R}=$ alkyl) and aryl-EBXs (3b, $\mathrm{R}=$ aryl) as coupling partners and thus truly delivers on the promise of affording unsymmetrical 1,3-butadiynes (75) having alkynes with even similar structures or electronic properties in high yields.

The reaction may follow two different pathways (cycle 1 or 2) to form common intermediate $\mathbf{8 1}$ based on the sequence of oxidation and transmetalation. A direct oxidation of $\mathbf{7 9}$ to $\mathbf{8 0}$, followed by coordination with the terminal alkyne, leads to the dialkynylgold(III) species $\mathbf{8 1}$ (cycle 1). Alternatively, the oxidation could occur preferentially on phen-ligated $\mathrm{Au}(\mathrm{I})$-acetylide $\mathbf{8 2}$ generated in situ, thus forming the common intermediate $\mathbf{8 1}$ (cycle 2). Once formed, the $\mathrm{Au}(\mathrm{III})$-species $\mathbf{8 1}$ undergoes reductive

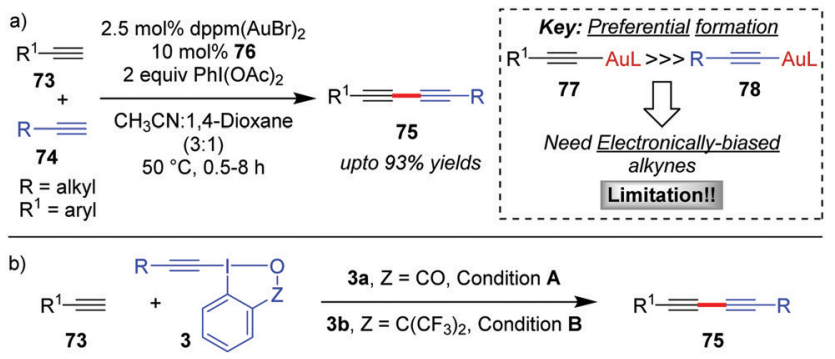

$\mathrm{R}=$ TIPS, TBDMS, TBPDS, aryl, ${ }^{n} \mathrm{Bu}$ $\mathrm{R}^{1}=$ alkyl, TMS, TIPS, aryl

Condition A: 3 mol\% $\mathrm{Ph}_{3} \mathrm{PAuCl}, 15 \mathrm{~mol} \% 76$ $\mathrm{CH}_{3} \mathrm{CN}: 1,4$-dioxane $(3: 1), 60^{\circ} \mathrm{C}$ 8-12 h, upto $86 \%$ yields (Patil's)

Condition B: $5 \mathrm{~mol} \%$ TA-Au, $5 \mathrm{~mol} \%$ AgOTs 0.5 equiv $76, \mathrm{CH}_{3} \mathrm{CN}$, $\mathrm{rt}$ upto $98 \%$ yields (Liu's)
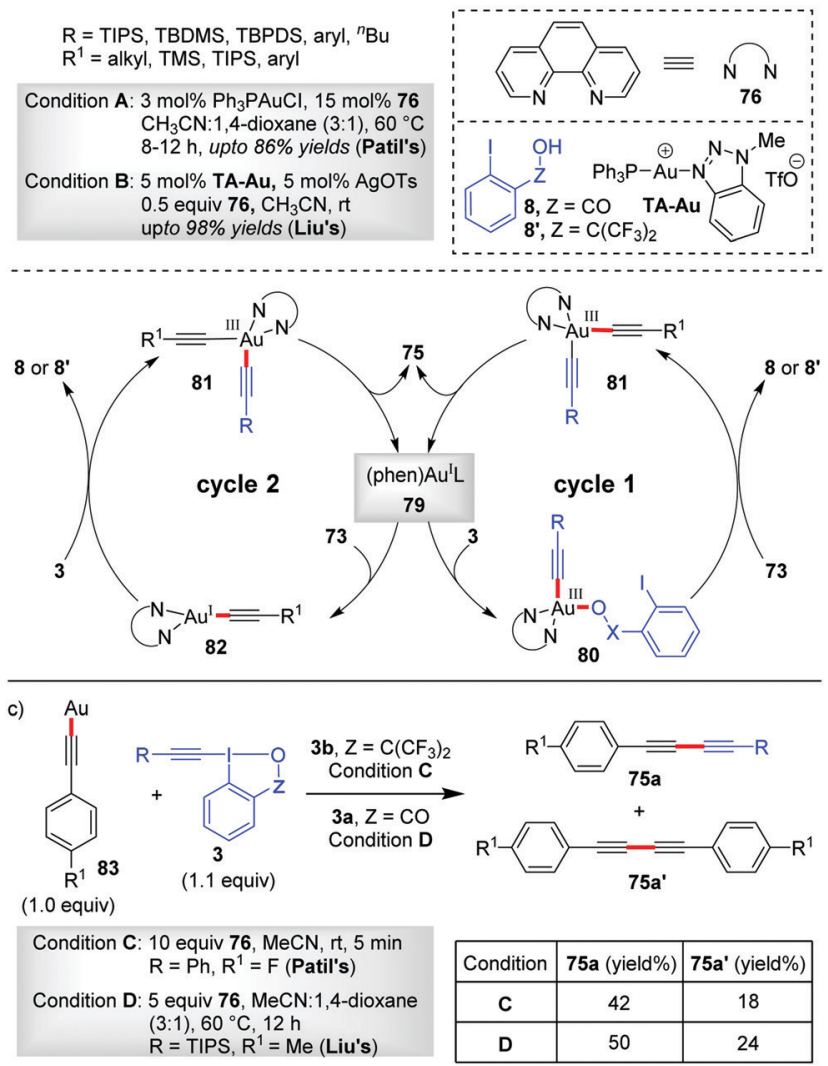

Scheme 15 Au-Catalyzed C(sp)-C(sp) cross-coupling reactions: (a) Shi's method; (b) Liu and Patil's method; and (c) control experiments with ligand-free polymeric Au(I)-acetylide by Liu and Patil.

elimination to give the desired cross-coupled product $\mathbf{7 5}$ with the regeneration of the active catalyst 79 . In a control experiment, the reaction involving a stoichiometric amount of the ligand-free polymeric Au-acetylide (83), which was supposed to generate $\mathbf{8 2}$ in situ under the standard reaction conditions, demonstrated commendable heteroselectivity (Scheme 15c). Apparently, it is suggested that the intermediacy of a phen-ligated acetylide (82) is highly likely and even the mechanistic pathway depicted in cycle 2 might be a favoured pathway over that of cycle 1 .

However, new mechanistic insights from Hashmi's group ${ }^{27 b}$ provided strong experimental evidence in favour of oxidative addition of 3 to the tri-coordinate $\mathrm{Au}(\mathrm{I})$-species $\mathbf{7 9}$ to generate 80, which essentially supports cycle 1 .

The Hashmi group in 2019 reported the direct alkynylation of cyclopropenes 84 via $\mathrm{Au} / \mathrm{Ag}$ cooperative-catalysis, ${ }^{38}$ affording alkynylsubstituted cyclopropenes (85, up to $92 \%$ yields, Scheme 16). ${ }^{27 a}$ The involvement of (phen) $\mathrm{Au}(\mathrm{I})^{(+)}(\mathbf{8 6})$ as the catalytically active 
species for all alkynylative cross-couplings with EBXs was widely speculated before. Using $\mathbf{9 4}$ as a pre-catalyst for $\mathbf{8 6}$, when the corresponding reaction rate was compared to that of other precatalysts $\left(\mathrm{PPh}_{3} \mathrm{AuNTf}_{2}\right.$ and $\left.\mathrm{AuCl}\right)$, it revealed a higher initial reaction rate in case of $\mathbf{9 4}$ which further confirmed the role of $\mathbf{8 6}$ as the active catalyst for the reaction. It is formed as a result of ligand exchange (between $\mathrm{PPh}_{3} \mathrm{AuNTf}_{2}$ and 76) at the beginning of the alkynylation reaction, which typically takes 20 minutes as per the observation of induction time for the alkynylation reaction. This ligand exchange process is notably similar to Liu and Patil's case as discussed in Scheme 15. Further, the essential role of $\mathrm{Ag}(\mathrm{I})^{(+)}$is manifested in the $\mathrm{C}-\mathrm{H}$ metalation of the cyclopropenes $\mathbf{8 4}$ rather than being a halogen scavenger as judged by $H / D$ exchange experiments with $\mathbf{8 4}$. The available mechanistic data indicate an oxidative catalytic cycle involving an alkynylgold(III) complex, $\mathbf{8 8}$, formed by the oxidative addition of the hypervalent iodine(III) reagent $3 \mathbf{b}$ to (phen) $\mathrm{Au}(\mathrm{I})^{(+)}(\mathbf{8 6})$. In fact, the putative alkynylgold(III) intermediate $\mathbf{8 8}$ was stoichiometrically generated in situ ( $c f$. 88a), characterized and engaged in a successful alkynylation of cyclopropene 84a, which further validated its intermediacy (Scheme 16b). After the formation of $\mathbf{8 8}$ in the mechanistic cycle, the alkoxy anion was then transferred from $\mathrm{Au}$ (III) to $\mathrm{Ag}$ (I) ( $c f$. 92), followed by $\mathrm{C}-\mathrm{H}$ metalation to afford the cyclopropenylsilver 93. Next, a transmetalation occurred between $\mathrm{Ag}(\mathrm{I})$ species 93 and $\mathrm{Au}(\mathrm{III})$-species 89 to obtain another $\mathrm{Au}(\mathrm{III})$-species, 90, followed by reductive elimination to form the alkynylated cyclopropene $\mathbf{8 5}$ with the regeneration of (phen)AuL 86. Recent mechanistic studies by the same group depicted a clearer picture of the formation of $\mathbf{8 8}$ which now consists of two steps of oxidative additions and one step of reductive elimination (Scheme 16c). ${ }^{27 b}$ The true identity of 86 is also revealed as (phen) $\mathrm{Ph}_{3} \mathrm{PAuNTf}_{2}$ (96), wherein the two $\mathrm{Au}-\mathrm{N}$ bonds are unsymmetric. The first oxidative addition to the unsymmetric tri-coordinate $\mathrm{Au}(\mathrm{I})$-species 96 proceeds with alkynyl hypervalent iodine $\mathbf{3 b}$ through a tetra-coordinate $\mathrm{Au}(\mathrm{I})$-transition state 97 (TS), affording 98. After that, carbon-phosphorus reductive elimination of $\mathrm{Au}(\mathrm{III})$-species (98) gives the trigonal $\mathrm{Au}(\mathrm{I})$-complex (100) along with 99, which has been observed experimentally. Finally, the $\mathrm{Au}(\mathrm{III})$-compound $\mathbf{8 8 a}$ forms after a second oxidative addition of $\mathbf{3 b}$ to $\mathbf{1 0 0}$.

\subsection{Alkynylations via 'interplay' mode}

According to our rationale B ( $c f$. Section 2), there exists a third mode of reactivity of Au-catalysts in alkynylation reactions with EBXs which involves the interplay of both the $\pi$-activation mode and $\mathrm{Au}(\mathrm{I}) / \mathrm{Au}(\mathrm{III})$ catalysis mode. The first instance of this 'interplay' mode was observed in the Waser group's report in 2013 wherein they established a domino cyclization/alkynylation approach to C3-alkynylated furans $\mathbf{1 0 4}$ (up to 97\% yields) from allenic ketones 102 and EBXs 3b in the presence of a $\mathrm{Au}(\mathrm{III})-$ catalyst, 105 (Scheme 17). ${ }^{20}$ This is indeed complementary to the C2-alkynylated furans $\mathbf{1 0 3}$ (up to 90\% yields), obtained via direct $\mathrm{C}-\mathrm{H}$ alkynylation of furans (101) with 3a and AuCl. Interestingly, they noticed that $\mathrm{AuCl}$ did not promote the former reaction at all, which is atypical of such alkynylation reactions. As the reaction mechanism was not understood yet,
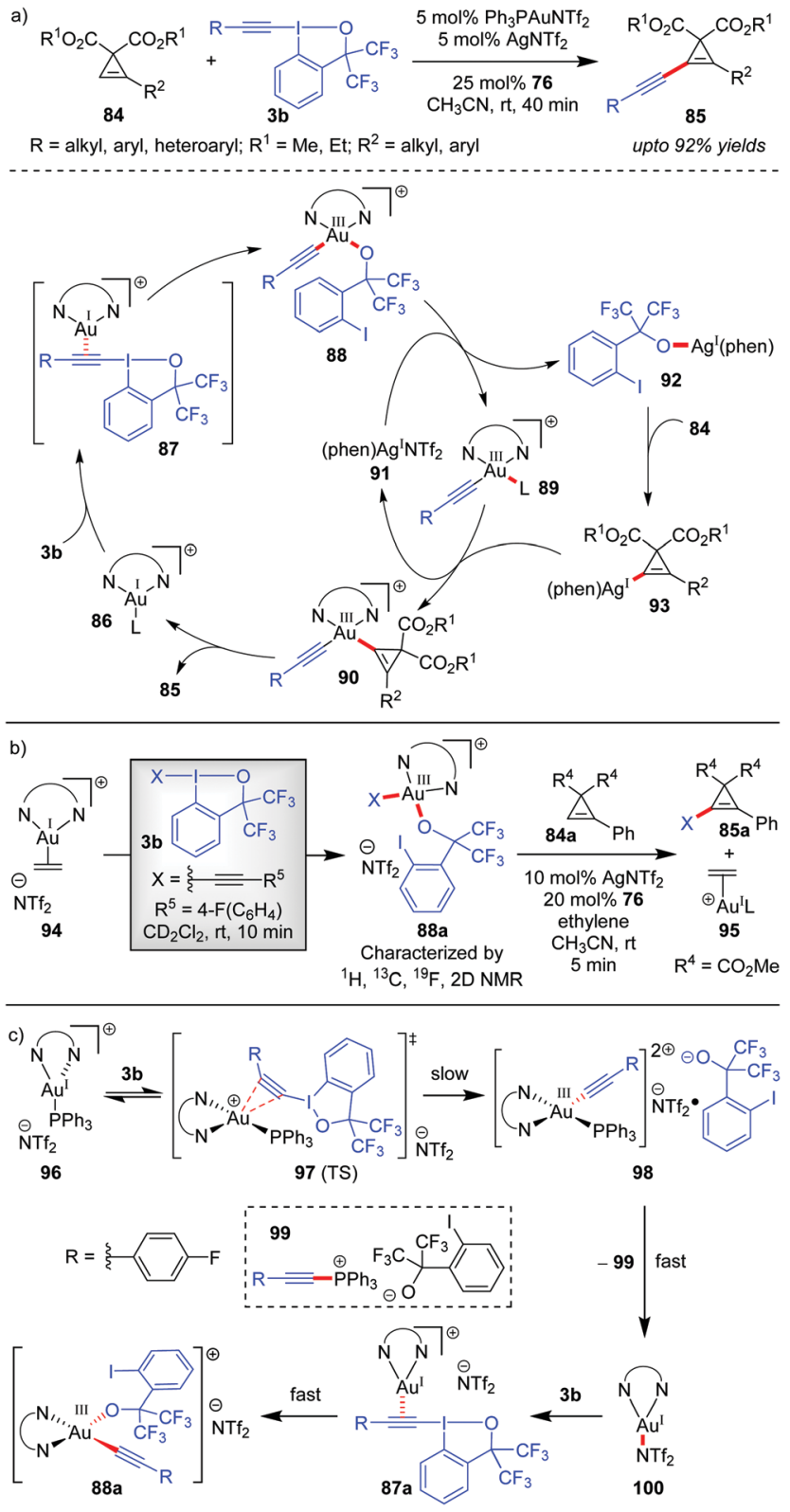

Scheme 16 (a) Direct alkynylation of cyclopropenes via Au/Ag cooperativecatalysis; (b) control experiment to prove (phen)Au(I) $)^{(+)}$as the active catalyst and the intermediacy of $\mathrm{Au}(\mathrm{II})$; and (c) new mechanistic insights into the formation of $\mathbf{8 8}$.

it was very difficult to rationalize the differences between the two catalytic processes (C3- vs. C2-alkynylation). In the new mechanistic insights provided by the Ariafard group, ${ }^{26}$ it was so disclosed that the used $\mathrm{Au}(\mathrm{III})$-catalyst $\mathbf{1 0 5}$ is a precursor of an active $\mathrm{Au}(\mathrm{I})$-catalyst, 107, which activates allene 102 towards 5-exo-dig cyclization. Thereafter, deprotonation gives the organogold(I) intermediate 109 which after oxidative addition of $\mathbf{3 b}$ offers 111 via transition state 110. Here, the DFT calculations further predict that the picolinic acid ligand (106) is responsible for stabilizing the $\mathrm{Au}(\mathrm{III})$-centre and thus essentially lowering the energy of the transition state 110. The resulting Au(III)-complex 111 then undergoes reductive elimination to give C3-alkynylated furans 

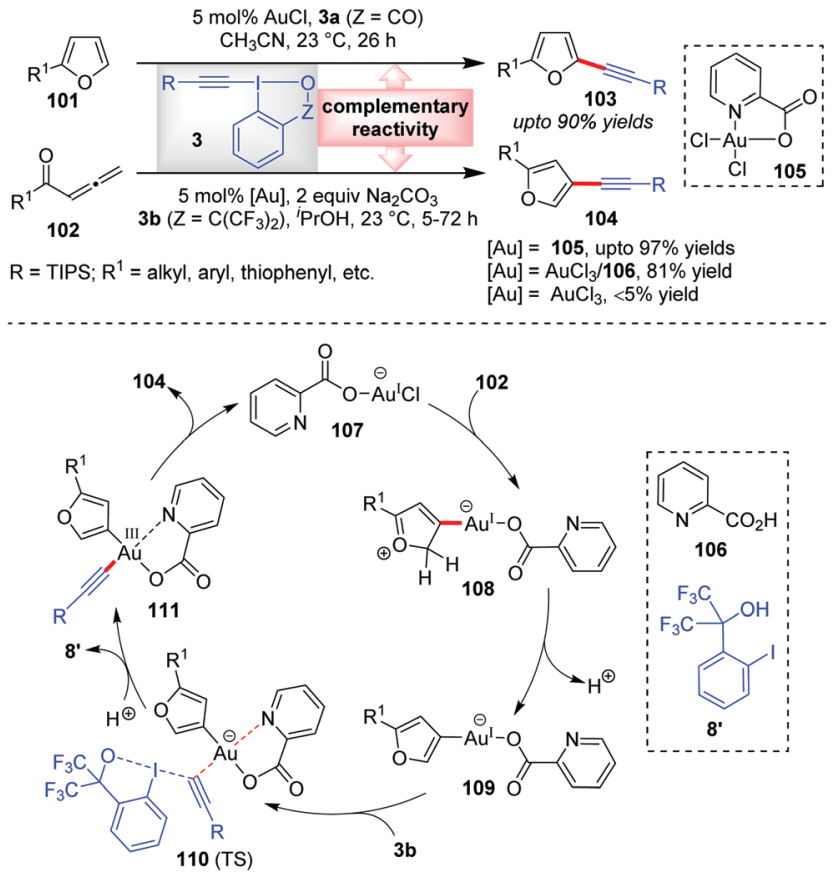

Scheme $17 \pi$-Activation mode and interplay mode of Au-catalysis giving rise to regioselective approaches towards C2- and C3-alkynyl furans, respectively.

104 and regenerates the active $\mathrm{Au}(\mathrm{I})$-catalyst 107. In contrast, the C2-alkynylation leading to $\mathbf{1 0 3}$ proceeds via the $\pi$-activation mode only (similar to cycle 1 , Scheme 2 ), as originally proposed by the authors. The complementary reactivity depicted here can be correlated with that of Scheme 13b, highlighting the unique alliance of Au-catalysis and EBXs in electrophilic alkynylation reactions.

Of note, control experiments regarding alternate catalyst systems reveal that the $\mathrm{AuCl}_{3} / \mathbf{1 0 6}$ catalyst system results in $81 \%$ yield, whereas bare $\mathrm{AuCl}_{3}$ gives less than $5 \%$ yield. This is similar to the observation reported by Huang's group in their $\alpha$ vinylidation ( $c f$. Scheme 7) that the performance of $\mathrm{AuCl}_{3}$ is significantly dependent on the presence of bidentate ligand $\mathbf{3 2}$ (31\% vs. 91\% yield). Similar is the case with AuCl (36\% vs. 93\% yield). The latter outcome may be rationalized in the way that 32 serves as a $\mathrm{Au}(\mathrm{III})$-stabilizing agent during the oxidative addition of $3 \mathbf{a}$ to $\mathrm{Au}(\mathrm{I})$, lowering the energy of the key transition state for oxidative addition. ${ }^{27,28}$ In the case of the former outcome with $\mathrm{AuCl}_{3}$, it is highly possible that the $\mathrm{Au}(\mathrm{III})$-complex, which forms in situ in coordination with ligand 32, is in fact a precursor of an active Au(I)-catalyst, in line with Ariafard's proposal, ${ }^{26}$ which requires the support of ligand $\mathbf{3 2}$ for oxidative addition. This leaves a reasonable speculation that the $\alpha$-vinylidation of aldehydes might proceed via a $\mathrm{Au}(\mathrm{I}) / \mathrm{Au}(\mathrm{III})$ catalysis mode as opposed to the $\pi$-activation mode proposed originally by Huang's group.

In another domino cyclization/alkynylation approach, where such interplay of activation modes is seen would be the highly stereoselective synthesis of $(E)$-alkynyloxazolines (113, up to $67 \%$ yields) by $N$-propargylcarboxamides 112 and benziodoxole
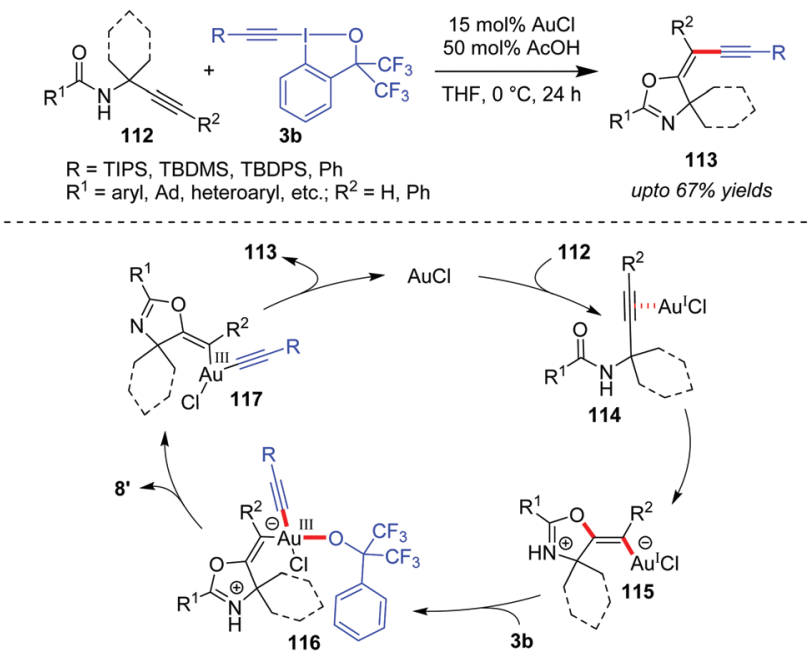

Scheme 18 Stereoselective domino cyclization/alkynylation of $\mathrm{N}$-propargylcarboxamides.

reagents $3 \mathbf{b}$ (Scheme 18). ${ }^{51}$ Mechanistically, the carbonyl oxygen atom attacks the alkyne, which is $\pi$-coordinated to $\mathrm{Au}(\mathrm{I})$, in a 5-exo-dig fashion to form vinylgold(I) intermediate 115. Next, the oxidative addition of intermediate $\mathbf{1 1 5}$ to $\mathbf{3 b}$ affords intermediate 116, which then undergoes ligand dissociation and reductive elimination to give the final product $(E)-\mathbf{1 1 3}$ with the regeneration of the active $\mathrm{Au}(\mathrm{I})$-catalyst. The authors suggested that the oxidative addition step is facilitated because intermediate $\mathbf{1 1 5}$ is a negatively charged ate-complex of $\mathrm{Au}(\mathrm{I})$. Further, a comparison of the computed energies of the $(Z)$ - and $(E)$-isomers of product 113 suggests kinetic control as the cause of the observed selectivity. Additionally, the acetic acid additive accelerates the catalytic cycle by protonating the alkoxide in complex 116, which assists the formation of $\mathbf{8}^{\prime}$. Notably, there are three such Au-catalyzed cyclization/alkynylation cascades with EBXs reported so far ( $c f$. Schemes 13, 17 and 18) and only Waser's work in Scheme 17 has received proper mechanistic investigation.

Despite their amazing forte of alkyne transfer, EBXs suffer a drawback in terms of the reactivity portfolio since 2-iodobenzoic acid (8) is generated as a stoichiometric byproduct. By introducing Cu-catalyzed oxyalkynylations of diazo compounds with $3 \mathbf{a}^{52}$ the Waser group resolved this issue by putting $\mathbf{8}$, the nucleophilic carboxylate part of EBXs, to appropriate use and became one of the pioneers in developing atom-economical ${ }^{53}$ use of 3a. Along similar lines, the Patil group recently disclosed the first Au-catalyzed 1,2-oxyalkynylation of $N$-allenamides (118) with appropriate atom-economical use of 3a, offering direct access to 1,3-enynes 119 (up to $83 \%$ yields, Scheme 19). ${ }^{28}$ Mechanistically, AuCl undergoes oxidative addition to EBXs (3a) to give the key tetracoordinated $\mathrm{Au}(\mathrm{III})$-species 120, which is stabilized with phen (76) and ion-paired with aryl carboxylate ligand 8. As an explanation of how the reaction takes place without a $\mathrm{Cl}^{(-)}$scavenger which is supposed to vacate a coordinating site of $\mathrm{Au}(\mathrm{III}),{ }^{22 a, 24 l, m}$ the authors surmised that the labile nature of bidentate ligand phen (76) allows the cationic $\mathrm{Au}(\mathrm{III})$ center of 120 to accommodate $\mathrm{N}$-allenamide (118) in its coordination sphere, leading to $\mathbf{1 2 1 .}$ 

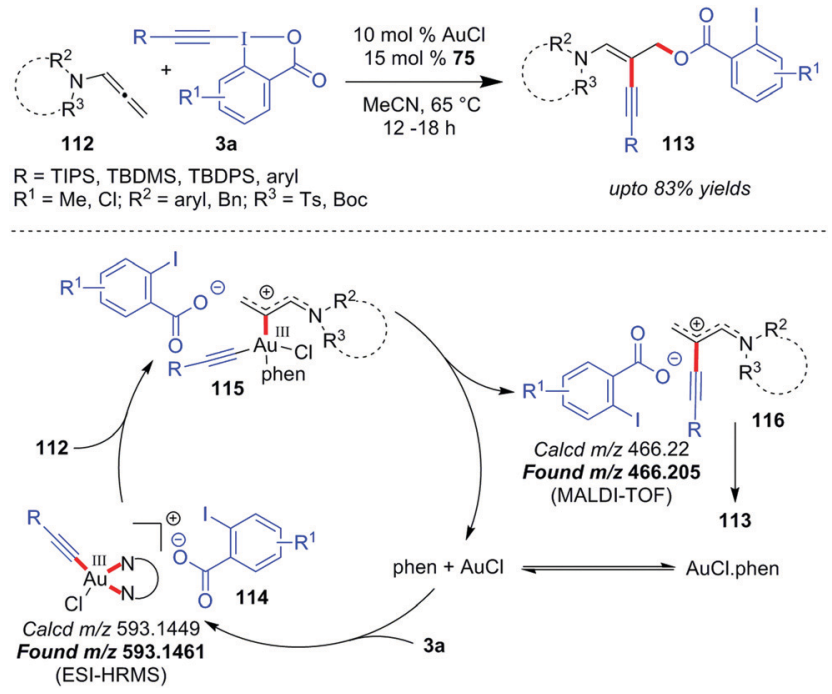

Scheme 19 1,2-Oxyalkynylation of $\mathrm{N}$-allenamides.

Thereafter, intermediate $\mathbf{1 2 1}$ upon reductive elimination affords species 122 and regenerates AuCl. Species 122 further undergoes conjugate attack by the laid-over carboxylate ligand 8 to afford the oxyalkynylated product 119. Both intermediates 120 and 122 are evidenced by tandem mass spectrometry analysis, which unequivocally supports a $\mathrm{Au}(\mathrm{I}) / \mathrm{Au}(\mathrm{III})$ catalysis mode.

Since the formation of allyl cationic $\mathrm{Au}(\mathrm{III})$-species 121 requires the carbophilic activation of $N$-allenamide 118 by species 120, this report constitutes another example of 'interplay' of activation modes for Au-catalyzed alkynylations. The detection of species $\mathbf{1 2 2}$ attests to the fact that reductive elimination precedes the conjugate nucleophilic attack of carboxylate anion 8 to ultimately provide the desired product. It is also interesting to notice that performing the title reaction without ligand phen (76) leaves no desirable outcome which, to some extent, resonates with the observations made by Huang ${ }^{37}$ and Hashmi. ${ }^{27 a}$

\section{Gold-catalyzed acetoxylations and arylations with hypervalent iodine(III) reagents}

Direct acetoxylation of arenes with transition metal catalysis, without employing the 'directing group' strategy, can become a challenging task because of regioselectivity issues. ${ }^{41 b}$ Based on the well-established fact that $\mathrm{Au}(\mathrm{III})$ can also electrophilically metalate an aromatic $\mathrm{C}-\mathrm{H}$ bond to generate arylgold(III) species, ${ }^{54}$ the Wang group was interested in investigating whether Au-catalyzed direct $\mathrm{C}-\mathrm{H}$ functionalization could become an alternative approach towards maintaining the regioselectivity, high efficiency and generality of the reaction. Towards this end, the Wang group developed a $\mathrm{AuCl}_{3}$-catalyzed direct acetoxylation of electron-rich aromatic compounds (123) with iodobenzene diacetate (1a) as the acetoxylation reagent to obtain 124 in high yields (up to $82 \%$, Scheme 20 ). ${ }^{55}$ In this case, the overacetoxylation problem (leading to di- and triacetoxylation products) has been
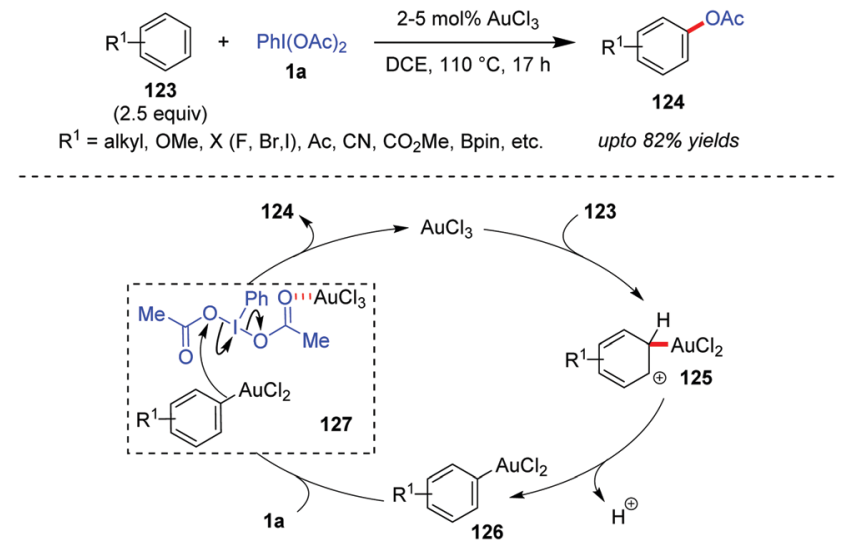

Scheme 20 Direct acetoxylation of arenes.

overcome by employing an excess amount (2.5 equiv.) of aromatic substrates. In the presence of a strong electron-withdrawing group $-\mathrm{CN}$ or $-\mathrm{CO}_{2} \mathrm{Me}$ as a substituent, a slightly higher catalyst loading (5\%) was required. The reaction also demonstrated commendable regioselectivity, which is evident from the fact that $123 a\left(R^{1}=\mathrm{OMe}\right)$ underwent para-acetoxylation only and in the case of other substituted anisole substrates, the methoxy substituent dictates the regioselectivity of the acetoxylation.

Mechanistically, it has been proposed that the arylgold(III) species 126 forms after a $\mathrm{C}-\mathrm{H}$ auration/deprotonation sequence (cf. 125) from 123. Thereafter, species 126 attacks the Au-activated iodobenzene diacetate ( $c f$. 127) to afford the acetoxylation product (124). Here the high catalytic efficiency of $\mathrm{AuCl}_{3}$ over other Lewis acid or protonic acid catalysts can be explained in terms of its dual activation mode of direct auration of $\mathbf{1 2 3}$ as well as the activation of 1a through complexation. Kinetic isotope effect studies show the formation of the Wheland intermediate 125 to be the rate-limiting step. An alternative mechanistic possibility in which an intermediate iodonium salt of the electron-rich aromatic substrate (123) could form and undergo a subsequent Au-mediated process leading to the final product (124) could not be ruled out by the Wang group.

The suitability of diaryliodonium salts (2a) as aryl sources is well-established in Pd- or Cu-catalyzed cross-coupling reactions. ${ }^{5 b, 56}$ In this context, the Glorius group described the first use of $2 \mathrm{a}$ as a coupling partner with a $\mathrm{Au} /$ photocatalyst system in the intermolecular multicomponent oxyarylation of simple, non-activated alkenes (128) with alcohol (129) under blue LEDs, leading to the desired arylated ether product $\mathbf{1 3 0}$ at room temperature (up to $91 \%$ yields, Scheme 21). ${ }^{57}$ The stability and ready accessibility of diaryliodonium salts (2a) over aryldiazonium salts makes them viable, alternative aryl-coupling partners in cross-coupling reactions using photoredox strategies. ${ }^{58}$ Mechanistically, coordination of the alkene (128) to the cationic $\mathrm{Au}(\mathrm{I})$-catalyst, followed by intermolecular nucleophilic attack of the alcohol (129), affords an alkylgold(I) complex of type 131. The aryl radical generated through SET reduction of a diaryliodonium salt (2a) by $\operatorname{Ir}(\mathrm{III})^{*}$ adds to complex 131 to furnish a $\mathrm{Au}(\mathrm{II})$-species of type 132, which is further oxidized by $\operatorname{Ir}(\mathrm{IV})$ to generate a $\mathrm{Au}(\mathrm{III})$-complex of type 133, with the completion of the photocatalytic cycle. 

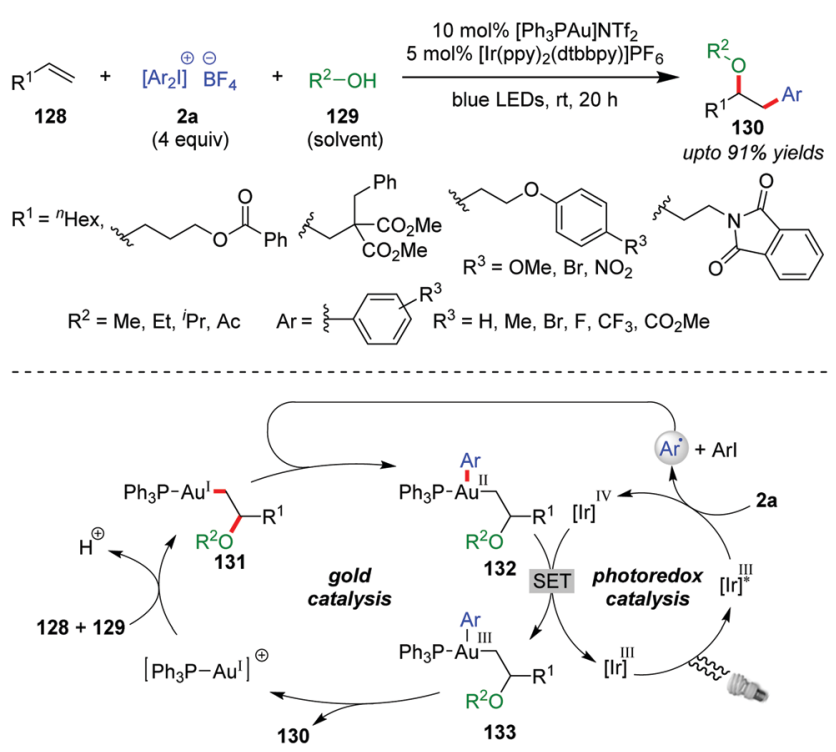

Scheme 21 Multicomponent oxyarylation of non-activated alkenes.

Finally, the $\mathrm{Au}(\mathrm{III})$-complex 133 undergoes reductive elimination to yield the product (130) with regeneration of the $\mathrm{Au}(\mathrm{I})$-catalyst. The use of Ir(III) as a photocatalyst over $\mathrm{Ru}(\mathrm{II})$ is preferred because excited state $\operatorname{Ir}(\mathrm{III})^{*}$ has a higher reduction potential than excited state $\mathrm{Ru}(\mathrm{II})$.

A single-step chemoselective arylsulfonylation of boronic acids (134) by potassium metabisulfite $\left(\mathrm{K}_{2} \mathrm{~S}_{2} \mathrm{O}_{5}, 135\right)$ and diaryliodonium salts (2a) to access substituted diarylsulfones (136) was accomplished by the Tu group under (NHC)Au(I)-catalysis (137) (up to $98 \%$ yields, Scheme 22$).{ }^{59}$ Even in the case of unsymmetrical diaryliodonium salts, the fact that a more electron-rich and bulky sulphone (containing $\mathrm{Ar}^{3}$ ) is formed over the less electron-rich and less bulky one (containing $\mathrm{Ar}^{2}$ ) showcases
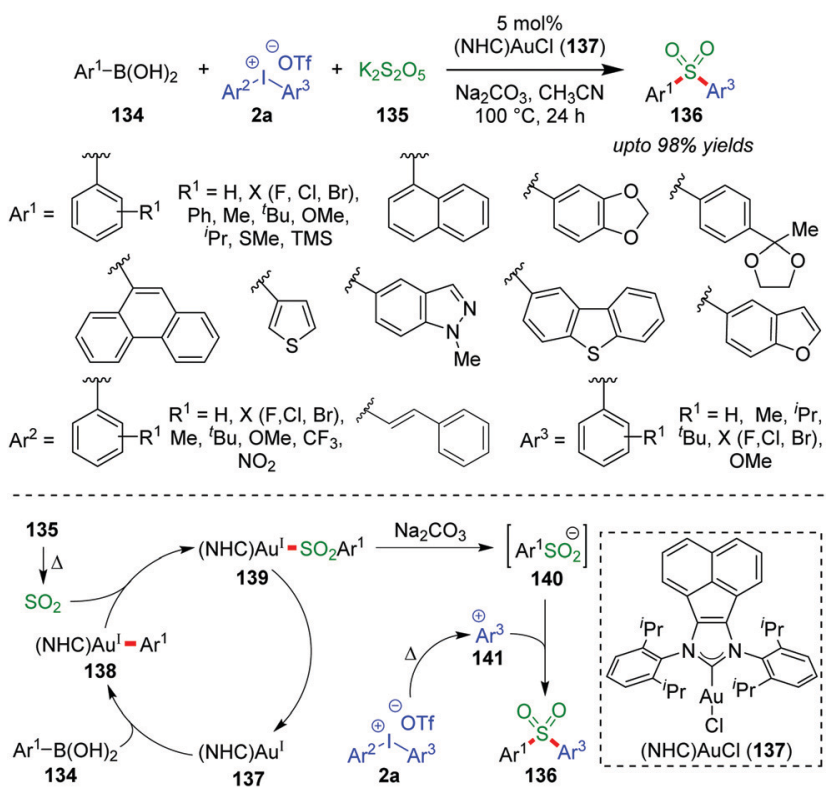

Scheme 22 Arylsulfonylation of boronic acids. the excellent chemoselectivity of the reaction. Mechanistically, this is explained on the basis of the stability of the intermediate (141) formed in situ from bulky and electron-rich aryl groups $\left(\mathrm{Ar}^{3}\right)$ over that of less bulky and electron-poor aryl groups $\left(\mathrm{Ar}^{2}\right)$. Meanwhile, sulfur dioxide, generated in situ by heating $\mathrm{K}_{2} \mathrm{~S}_{2} \mathrm{O}_{5}$, is inserted into the $\mathrm{Au}-\mathrm{C}_{\mathrm{Ar}}$ bond of intermediate 138, which has resulted from an initial transmetalation of $\mathbf{1 3 7}$ with aryl boronic acid (134). Consequently, sterically congested sulfonyl $\mathrm{Au}(\mathrm{I})$ complex 139 produces the key sulfinate intermediate 140 and regenerates the $\mathrm{NHC}-\mathrm{Au}(\mathrm{I})$-species $\mathbf{1 3 7}$ under basic conditions. The crucial sulphinate intermediate $\mathbf{1 4 0}$ can react with the more stable, bulky and electron-rich $\mathbf{1 4 1}$ formed from diaryliodonium salts (2a) and thus afford the diarylsulfones (136). The current selectivity is in direct contrast to Pd-catalyzed $\mathrm{C}-\mathrm{C}_{\mathrm{Ar}}$ bond formation reactions, in which the less bulky aryl groups are usually transferred. ${ }^{60}$

\section{Conclusions and future outlook}

From just being a passing infatuation for chemists for their unique structural characteristics to becoming well-established reagents in mainstream synthetic chemistry ensuring delivery of functional groups with reversed polarity, hypervalent iodine(III) reagents have come a long way. The exceptional reactivity of hypervalent iodine(III) reagents, in combination with the extra stability and modularity of the cyclic system, is primarily behind their popularity amongst the scientific community. If we closely scrutinize the development of such functional group transfer reactions with hypervalent iodine(III) reagents, especially alkynylations with EBXs, we are bound to find an undeniable correlation with the development of Au-catalysis. With their two different modes of activation (i.e. $\pi$-activation mode or $\mathrm{Au}(\mathrm{I}) /$ $\mathrm{Au}(\mathrm{III})$ catalysis mode), which can also interplay under certain conditions ('interplay mode'), Au-catalysts have formed a strong liaison with EBXs to achieve a wide array of alkynylations. Mechanistically, it is now understood that in the presence of a stabilizing agent in the reaction, which is often bidentate $\mathrm{N}$-coordinating ligands like phen (76) or picolinic acid (106), the alkynylation reaction is more likely to proceed via $\mathrm{Au}(\mathrm{I}) / \mathrm{Au}(\mathrm{III})$ catalysis. Despite this, in some reports where a mechanistic ambiguity still exists as described by the authors, the intervention of computational chemists to gain proper insights into the mechanism is necessary. Personally, we believe that the mechanism for gold-catalyzed alkynylations with EBXs would vary depending on the reaction.

It is, however, true that the number of gold-catalysts that are well-established with hypervalent iodine(III) reagents concerning functional group transfer reactions is limited. In such cases, tuning the reactivity of hypervalent iodine(III) reagents via modulation of the 'ligand-sphere' around the iodine atom can be one of the solutions (e.g. 3a vs. 3b) which was elegantly utilized by Waser (Scheme 17). In fact, many variants of such cyclic $\lambda^{3}$-alkynyliodanes have been reported so far with 'tuned' reactivity ${ }^{7 b, 52 c}$ and are waiting to be screened with gold-catalysts. Furthermore, enantioselective electrophilic alkynylations with EBXs under Au-catalysis would be a 
much-anticipated accomplishment in the near future. In particular, conducting the alkynylation of alkenes/allenes and the $\mathrm{C}\left(\mathrm{sp}^{3}\right)-\mathrm{H}$ alkynylations of unactivated systems in regio-, diastereo- and enantioselective manners would be particularly desirable for future applications of EBXs. ${ }^{61}$ One way to achieve this is to incorporate a suitable combination of a $\mathrm{Au}(\mathrm{I}) / \mathrm{chiral}$ hypervalent iodine(III) ${ }^{62}$ system in the transformation. Alternatively, since hypervalent iodine(III) reagents can oxidize $\mathrm{Au}(\mathrm{I})$ to $\mathrm{Au}(\mathrm{III})$ in situ and chiral $\mathrm{Au}(\mathrm{III})$-complexes ${ }^{63}$ can effect better chiral induction than $\mathrm{Au}(\mathrm{I})$-complexes, the chiral $\mathrm{Au}(\mathrm{I}) /$ hypervalent iodine(III) system can also be explored along this line. On the other hand, while the report on the progress of Au-catalyzed electrophilic alkynylation over the past decade is impressive and noteworthy, the available literature on other such Au-catalyzed functional group transfer processes such as arylation ${ }^{56}$ and trifluoromethylation ${ }^{6 b}$ is still trailing behind. In particular, in arylations under gold/photoredox catalysis, the potential of diaryliodonium salts (2a) as alternative aryl-sources hasn't been exploited much. Nevertheless, as a consequence of the strong alliance between the $\pi$-activation capability of Au-catalysts and the dual-functioning capability of EBXs as alkyne coupling partners as well as oxidants that can effortlessly overcome the high oxidation potential of the $\mathrm{Au}(\mathrm{I}) / \mathrm{Au}(\mathrm{III})$ redox couple, ${ }^{64}$ the Au-catalyzed cross-coupling reactions are currently undergoing a paradigm shift in terms of generating new reactivities and selectivities under redoxneutral conditions. Therefore, the authors strongly believe that a paradigm shift awaits us where Au-catalysts, with their different modes of activation as discussed in the current article, are going to make remarkable contribution to promoting new reactivities (e.g. Au-carbenes with cyclic $\lambda^{3}$-alkynyliodanes), which would further underscore the liaison of Au-catalysts with hypervalent iodine(III) reagents in electrophilic functional group transfer reactions.

\section{Conflicts of interest}

There are no conflicts to declare.

\section{Acknowledgements}

Generous financial support by the Science and Engineering Research Board (SERB), New Delhi (File No. EMR/2016/007177 and DIA/2018/000016), is gratefully acknowledged for related research in our laboratory. SB and VWB thank UGC and CSIR, respectively, for providing Senior Research Fellowships (SRF).

\section{Notes and references}

1 (a) Iodine Chemistry and Applications, ed. T. Kaiho, John Wiley \& Sons, Inc., New York, 2015; (b) V. V. Zhdankin, Hypervalent Iodine Chemistry: Preparation, Structure and Synthetic Application of Polyvalent Iodine Compounds, John Wiley \& Sons Ltd., New York, 2014; (c) M. Ochiai, in Hypervalent Iodine Chemistry: Modern Developments in Organic Synthesis, ed. T. Wirth, Springer, New York, 2003; (d) Chemistry of Hypervalent Compounds, ed. K. Y. Akiba, Wiley-VCH, New York, 1999. Selected reviews on the application of hypervalent iodine(III) reagents; (e) X. Wang and A. Studer, Acc. Chem. Res., 2017, 50, 1712; $(f)$ A. Yoshimura and V. V. Zhdankin, Chem. Rev., 2016, 116, 3328; (g) L. Wang and J. Liu, Eur. J. Org. Chem., 2016, 1813; (h) V. V. Zhdankin and P. J. Stang, Chem. Rev., 2008, 108, 5299; $(i)$ T. Wirth, Angew. Chem., Int. Ed., 2005, 44, 3656; ( $j$ ) V. V. Zhdankin, Curr. Org. Synth., 2005, 2, 121; (k) V. V. Zhdankin, Rev. Heteroat. Chem., 1997, $17,133$.

2 Although the concept of hypervalency of polycoordinated maingroup elements like iodine has been the subject of controversy, it has been widely accepted by synthetic chemists to describe the structural features and special reactivity of such elements. See: R. J. Gillespie and B. Silvi, Chem. Rev., 2002, 233-234, 53.

3 J. P. Brand, D. F. González, S. Nicolai and J. Waser, Chem. Commun., 2011, 47, 102.

4 (a) F. M. Beringer and S. A. Galton, J. Org. Chem., 1965, 30, 1930; (b) F. M. Beringer, P. S. Forgione and M. D. Yudis, Tetrahedron, 1960, $8,49$.

5 (a) J. P. Brand and J. Waser, Chem. Soc. Rev., 2012, 41, 4165; (b) E. A. Merritt and B. Olofsson, Angew. Chem., Int. Ed., 2009, 48, 9052.

6 (a) D. P. Hari, S. Nicolai and J. Waser, Patai's Chemistry of Functional Groups, John Wiley \& Sons, Ltd, Chichester, UK, 2018, DOI: 10.1002/ 9780470682531.pat0951; (b) D. Katayev, B. Jelier and A. Togni, Patai's Chemistry of Functional Groups, John Wiley \& Sons, Ltd, Chichester, UK, 2018, DOI: 10.1002/9780470682531.pat0949.

7 For general reviews on EBXs, see: $(a)$ D. P. Hari, P. Caramenti and J. Waser, Acc. Chem. Res., 2018, 51, 3212; (b) J. Waser, Synlett, 2016, 2761; (c) Y. Li, D. P. Hari, M. V. Vita and J. Waser, Angew. Chem., Int. Ed., 2016, 55, 4436; (d) J. Kaschel and D. B. Werz, Angew. Chem., Int. Ed., 2015, 54, 8876.

8 M. Ochiai, Y. Masaki and M. Shiro, J. Org. Chem, 1991, 56, 5511.

9 R. L. Amey and J. C. Martin, J. Org. Chem., 1979, 44, 1779.

10 V. V. Zhdankin, C. J. Kuehl, A. P. Krasutsky, J. T. Bolz and A. J. Simonsen, J. Org. Chem., 1996, 61, 6547.

11 J. Charpentier, N. Früh and A. Togni, Chem. Rev., 2015, 115, 650.

12 J. P. Brand, J. Charpentier and J. Waser, Angew. Chem., Int. Ed., 2009, 48, 9346.

13 J. P. Brand, C. Chevalley, R. Scopelliti and J. Waser, Chem. - Eur. J., 2012, 18, 5655.

14 G. L. Tolnai, Z. Gonda and Z. Novák, Patai's Chemistry of Functional Groups, John Wiley \& Sons, Ltd., Chichester, UK, 2018, DOI: 10.1002/ 9780470682531.pat0961.

15 (a) Gold Catalysis: An Homogeneous Approach (Catalytic Science Series), ed. F. D. Toste and M. Veronique, Imperial College Press, Singapore, 2014, vol. 13; (b) Modern Gold Catalyzed Synthesis, ed. A. S. K. Hashmi and F. D. Toste, Wiley-VCH, 2012.

16 For conceptual reviews on the superiority of gold-catalysis, see: (a) D. J. Gorin and F. D. Toste, Nature, 2007, 446, 395; (b) A. Fürstner and P. W. Davies, Angew. Chem., Int. Ed., 2007, 46, 3410. For general reviews on gold-catalysis, see: $(c)$ R. Dorel and A. M. Echavarren, Chem. Rev., 2015, 115, 9028; (d) N. T. Patil, Curr. Sci., 2013, 104, 1671; (e) A. Corma, A. Leyva-Pérez and M. J. Sabater, Chem. Rev., 2011, 111, 1657; $(f)$ M. Bandini, Chem. Soc. Rev., 2011, 40, 1358; (g) T. C. Boorman and I. Larrosa, Chem. Soc. Rev., 2011, 40, 1910; (h) M. Rudolph and A. S. K. Hashmi, Chem. Commun., 2011, 47, 6536; (i) E. Jiménez-Núñez and A. M. Echavarren, Chem. Rev., 2008, 108, 3326; $(j)$ Z. Li, C. Brouwer and C. He, Chem. Rev., 2008, 108, 3239; (k) A. S. K. Hashmi and M. Rudolph, Chem. Soc. Rev., 2008, 37, 1766; (l) A. Arcadi, Chem. Rev., 2008, 108, 3266; $(m)$ A. S. K. Hashmi and G. J. Hutchings, Angew. Chem., Int. Ed., 2006, 45, 7896; (n) A. S. K. Hashmi and G. J. Hutchings, Angew. Chem., Int. Ed., 2006, 45, 7896.

17 The idea was greatly inspired by the pioneering work of Sanford and Gaunt on the arylation of indoles with aryliodonium salts. (a) R. J. Phipps, N. P. Grimster and M. J. Gaunt, J. Am. Chem. Soc., 2008, 130, 8172; (b) N. R. Deprez, D. Kalyani, A. Krause and M. S. Sanford, J. Am. Chem. Soc., 2006, 128, 4972. During this investigation, palladium catalysts gave only traces of C3 alkynylated indoles, albeit with very high C2 selectivity, see: (c) G. L. Tolnai, S. Ganss, J. P. Brand and J. Waser, Org. Lett., 2013, 15, 112.

18 J. P. Brand and J. Waser, Angew. Chem., Int. Ed., 2010, 49, 7304.

19 J. P. Brand and J. Waser, Org. Lett., 2012, 14, 744.

20 Y. Li, J. P. Brand and J. Waser, Angew. Chem., Int. Ed., 2013, 52, 6743. 21 Y. Li and J. Waser, Beilstein J. Org. Chem., 2013, 9, 1763.

22 Recent reports on gold catalysis from our lab: (a) M. O. Akram, A. Das, I. Chakrabarty and N. T. Patil, Org. Lett., 2019, 21, 8101; (b) C. C. Chintawar, M. V. Mane, A. G. Tathe, S. Biswas and N. T. Patil, 
Org. Lett., 2019, 21, 7109; (c) R. D. Mule, A. C. Shaikh, A. B. Gade and N. T. Patil, Chem. Commun., 2018, 54, 11909; (d) I. Chakrabarty, M. O. Akram, S. Biswas and N. T. Patil, Chem. Commun., 2018, 54, 7223; (e) A. C. Shaikh, D. S. Ranade, P. R. Rajamohanan, P. P. Kulkarni and N. T. Patil, Angew. Chem., Int. Ed., 2017, 56, 757; $(f)$ M. O. Akram, P. S. Mali and N. T. Patil, Org. Lett., 2017, 19, 3075; $(g)$ P. N. Bagle, M. V. Mane, K. Vanka, D. R. Shinde, S. R. Shaikh, R. G. Gonnade and N. T. Patil, Chem. Commun., 2016, 52, 14462; $(h)$ A. B. Gade and N. T. Patil, Org. Lett., 2016, 18, 1844.

23 For an overview on gold catalysis in cross-coupling reactions, see: (a) A. Nijamudheen and A. Datta, Chem. - Eur. J., 2020, 26, 1442; (b) M. Livendahl and A. M. Echavarren, Chim. Oggi, 2012, 30, 19.

24 For reviews on sacrificial oxidant-aided oxidative gold catalysis, see: (a) K. Chen and S. Zhu, Synlett, 2017, 640; (b) S. Kramer, Chem. - Eur. J., 2016, 22, 1558; (c) Z. Zheng, Z. Wang, Y. Wang and L. Zhang, Chem. Soc. Rev., 2016, 45, 4448; (d) J. Miro and C. del Pozo, Chem. Rev., 2016, 116, 11924; (e) K. M. Engle, T.-S. Mei, X. Wang and J.-Q. Yu, Angew. Chem., Int. Ed., 2011, 50, 1478; $(f)$ H. A. Wegner and M. Auzias, Angew. Chem., Int. Ed., 2011, 50, 8236; $(g)$ M. N. Hopkinson, A. D. Gee and V. Gouverneur, Chem. - Eur. J., 2011, 17, 8248; (h) P. Garcia, M. Malacria, C. Aubert, V. Gandon and L. Fensterbank, ChemCatChem, 2010, 2, 493. For reviews on oxidant-free oxidative gold catalysis, see: (i) M. O. Akram, S. Banerjee, S. S. Saswade, V. Bedi and N. T. Patil, Chem. Commun., 2018, 54, 11069. For reviews on oxidative gold-catalysis by employing a visible-light photoredox strategy, see: $(j) \mathrm{J}$. Xie, H. Jin and A. S. K. Hashmi, Chem. Soc. Rev., 2017, 46, 5193; (k) M. N. Hopkinson, A. Tlahuext-Aca and F. Glorius, Acc. Chem. Res., 2016, 49, 2261. For reports on oxidative gold catalysis effected by rational ligand designs, see: (l) J. Rodriguez, N. Adet, N. Saffon-Merceron and D. Bourissou, Chem. Commun., 2020, 56, 94; (m) A. Zeineddine, L. Estévez, S. Mallet-Ladeira, K. Miqueu, A. Amgoune and D. Bourissou, Nat. Commun., 2017, 8, 565. 25 A. Ariafard, ACS Catal., 2014, 4, 2896.

26 H. Ghari, Y. Li, R. Roohzadeh, P. Caramenti, J. Waser and A. Ariafard, Dalton Trans., 2017, 46, 12257.

27 (a) Y. Yang, P. Antoni, M. Zimmer, K. Sekine, F. Mulks, L. Hu, L. Zhang, M. Rudolph, F. Rominger and A. S. K. Hashmi, Angew. Chem., Int. Ed., 2019, 58, 5129; (b) Y. Yang, L. Eberle, F. F. Mulks, J. F. Wunsch, M. Zimmer, F. Rominger, M. Rudolph and A. S. K. Hashmi, J. Am. Chem. Soc, 2019, 141, 17414.

28 S. Banerjee, B. Senthilkumar and N. T. Patil, Org. Lett., 2019, 21, 180.

29 G. N. Hermann, M. T. Unruh, S.-H. Jung, M. Krings and C. Bolm, Angew. Chem., Int. Ed., 2018, 57, 10723.

30 J. P. Brand, C. Chevalley and J. Waser, Beilstein J. Org. Chem., 2011, 7, 565.

31 (a) P. K. Sajith and C. H. Suresh, Inorg. Chem., 2013, 52, 6046; (b) P. K. Sajith and C. H. Suresh, Inorg. Chem., 2012, 51, 967; (c) M. Ochiai, T. Sueda, K. Miyamoto, P. Kiprof and V. V. Zhdankin, Angew. Chem., Int. Ed., 2006, 45, 8203.

32 G. L. Tolnai, J. P. Brand and J. Waser, Beilstein J. Org. Chem., 2016, $12,745$.

33 A. C. Shaikh, D. R. Shinde and N. T. Patil, Org. Lett., 2016, 18, 1056.

34 Y. Li, F. Xie and X. Li, J. Org. Chem., 2016, 81, 715.

35 F. Zhao, B. Xu, D. Ren, L. Han, Z. Yu and T. Liu, Organometallics, 2018, 37, 1026.

36 M. O. Akram, S. Bera and N. T. Patil, Chem. Commun., 2016, 52, 12306.

37 Z. Wang, X. Li and Y. Huang, Angew. Chem., Int. Ed., 2013, 52, 14219.

38 N. T. Patil, V. S. Shinde and B. Gajula, Org. Biomol. Chem., 2012, 10, 211.

39 Z. Wang, L. Li and Y. Huang, J. Am. Chem. Soc., 2014, 136, 12233.

40 S. Peng, Z. Wang, L. Zhang, X. Zhang and Y. Huang, Nat. Commun., $2018,9,375$.

41 (a) M. Tobisu, Y. Ano and N. Chatani, Org. Lett., 2009, 11, 3250. For a general review on palladium-catalyzed ligand-directed $\mathrm{C}-\mathrm{H}$ functionalization, see: $(b)$ T. W. Lyons and M. S. Sanford, Chem. Rev., 2010, 110, 1147.

42 A. Székely, Á. Péter, K. Aradi, G. L. Tolnai and Z. Novák, Org. Lett., 2017, 19, 954.

43 P. S. Shinde and N. T. Patil, Eur. J. Org. Chem., 2017, 3512.

44 P. S. Shinde, A. C. Shaikh and N. T. Patil, Chem. Commun., 2016, 52, 8152. A relatable Pt(II)-catalyzed version can be found is Waser's report, see: Y. Li and J. Waser, Angew. Chem., Int. Ed., 2015, 54, 5438.

45 Selected reports: (a) X. C. Cambeiro, N. Ahlsten and I. Larrosa, J. Am. Chem. Soc., 2015, 137, 15636; (b) L. T. Ball, G. C. Lloyd-Jones and C. A. Russell, Science, 2012, 337, 1644; (c) T. de Haro and C. Nevado, J. Am. Chem. Soc., 2010, 132, 1512.

46 X. Wang, X. Li, Y. Zhang and L. Xia, Org. Biomol. Chem., 2018, 16, 2860. 47 (a) X. Li, X. Xin, N. Sun and Y. Liu, Angew. Chem., Int. Ed., 2017, 56, 6994; (b) S. Banerjee and N. T. Patil, Chem. Commun., 2017, 53, 7937.

48 H. Peng, Y. Xi, N. Ronaghi, B. Dong, N. G. Akhmedov and X. Shi, J. Am. Chem. Soc., 2014, 136, 13174.

49 (a) A. S. Hay, J. Org. Chem., 1962, 27, 3320; (b) A. S. Hay, J. Org. Chem., 1960, 25, 1275; (c) C. Glaser, Ber. Dtsch. Chem. Ges., 1869, $2,422$.

50 W. Chodkiewicz and P. Cadiot, Compt. Rend., 1955, 241, 1055.

51 X. Zhao, B. Tian, Y. Yang, X. Si, F. F. Mulks, M. Rudolph, F. Rominger and A. S. K. Hashmi, Adv. Synth. Catal., 2019, 361, 3155.

52 (a) D. P. Hari and J. Waser, J. Am. Chem. Soc, 2017, 139, 8420; (b) D. P. Hari and J. Waser, J. Am. Chem. Soc., 2016, 138, 2190. For a recent report on oxyalkynylation of $\mathrm{C}-\mathrm{S}$ bonds, see: $(c) \mathrm{J}$. Borrel, G. Pisella and J. Waser, Org. Lett., 2020, 22, 422. For a related reaction with ethynylbenziodazolones (EBZs), see: (d) D. P. Hari, L. Schouwey, V. Barber, R. Scopelliti, F. Fadaei-Tirani and J. Waser, Chem. - Eur. J., 2019, 25, 9522.

53 Atom-economical use of 3a was originally discovered by Yoshikai's group: (a) J. Wu and N. Yoshikai, Angew. Chem., Int. Ed., 2015, 54, 11107; (b) B. Lu, J. Wu and N. Yoshikai, J. Am. Chem. Soc., 2014, 136, 11598.

54 (a) Y. Fuchita, Y. Utsunomiya and M. Yasutake, J. Chem. Soc., Dalton Trans., 2001, 2330; (b) P. W. de Graaf, J. Boersma and G. J. M. van der Kerk, J. Organomet. Chem., 1976, 105, 399; (c) K. S. Liddle and C. Perkin, J. Chem. Soc., Chem. Commun., 1972, 26; (d) M. S. Kharasch and H. S. Isbell, J. Am. Chem. Soc., 1931, 53, 3053.

55 D. Qiu, Z. Zheng, F. Mo, Q. Xiao, Y. Tian, Y. Zhang and J. Wang, Org. Lett., 2011, 13, 4988.

56 (a) B. Olofsson, in Topics in Current Chemistry, ed. T. Wirth, Springer International Publishing, 2016, 373, 135; (b) P. Villo and B. Olofsson, in Patai's Chemistry of Functional Groups: The Chemistry of Hypervalent Halogen Compounds, ed. B. Olofsson, I. Marek, Z. Rappoport, 2018, DOI: 10.1002/9780470682531.pat0950.

57 M. N. Hopkinson, B. Sahoo and F. Glorius, Adv. Synth. Catal., 2014, 356, 2794.

58 K. Jia and Y. Chen, Patai's Chemistry of Functional Groups, John Wiley \& Sons, Ltd., Chichester, UK, 2018, DOI: 10.1002/9780470682531.pat0958.

59 H. Zhu, Y. Shen, D. Wen, Z.-G. Le and T. Tu, Org. Lett., 2019, 21, 974.

60 D. Kalyani, N. R. Deprez, L. V. Desai and M. S. Sanford, J. Am. Chem. Soc., 2005, 127, 7330.

61 J. Xie, S. Shi, T. Zhang, N. Mehrkens, M. Rudolph and A. S. K. Hashmi, Angew. Chem., Int. Ed., 2015, 54, 6046.

62 Recent reviews on chiral hypervalent iodine(III) reagents: (a) A. Parra, Chem. Rev., 2019, 119, 12033; (b) H. Liang and M. A. Ciufolini, Angew. Chem., Int. Ed., 2011, 50, 11849; (c) A. Parra and S. Reboredo, Chem. - Eur. J, 2013, 19, 17244.

63 (a) J.-J. Jiang, J.-F. Cui, B. Yang, Y. Ning, N. C.-H. Lai and M.-K. Wong, Org. Lett., 2019, 21, 6289; (b) P. T. Bohan and F. D. Toste, J. Am. Chem. Soc., 2017, 139, 11016.

64 S. G. Bratsch, J. Phys. Chem. Ref. Data, 1989, $18,1$. 